\title{
Study of $Z$ boson plus jets events using variables sensitive to double-parton scattering in pp collisions at $13 \mathrm{TeV}$
}

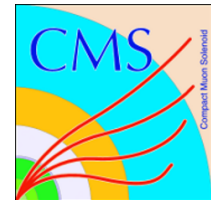

\section{The CMS collaboration}

E-mail: cms-publication-committee-chair@cern.ch

ABSTRACT: Double-parton scattering is investigated using events with a $\mathrm{Z}$ boson and jets. The $\mathrm{Z}$ boson is reconstructed using only the dimuon channel. The measurements are performed with proton-proton collision data recorded by the CMS experiment at the LHC at $\sqrt{s}=13 \mathrm{TeV}$, corresponding to an integrated luminosity of $35.9 \mathrm{fb}^{-1}$ collected in the year 2016. Differential cross sections of $\mathrm{Z}+\geq 1$ jet and $\mathrm{Z}+\geq 2$ jets are measured with transverse momentum of the jets above $20 \mathrm{GeV}$ and pseudorapidity $|\eta|<2.4$. Several distributions with sensitivity to double-parton scattering effects are measured as functions of the angle and the transverse momentum imbalance between the $\mathrm{Z}$ boson and the jets. The measured distributions are compared with predictions from several event generators with different hadronization models and different parameter settings for multiparton interactions. The measured distributions show a dependence on the hadronization and multiparton interaction simulation parameters, and are important input for future improvements of the simulations.

KEYwORDS: Hadron-Hadron scattering (experiments), Jets

ArXiv EPrint: 2105.14511 


\section{Contents}

1 Introduction 1

2 The CMS detector 3

3 Simulated event samples 3

4 Event selection $\quad 5$

$\begin{array}{lll}5 & \text { Observables and the unfolding } & 7\end{array}$

6 Systematic uncertainties $\quad 9$

$\begin{array}{lll}7 & \text { Results } & 10\end{array}$

$\begin{array}{lll}8 & \text { Summary } & 14\end{array}$

$\begin{array}{ll}\text { The CMS collaboration } & 23\end{array}$

\section{Introduction}

Proton-proton ( $\mathrm{pp}$ ) collisions at high energies result in many events with a $\mathrm{Z}$ boson produced in association with jets at large transverse momentum $p_{\mathrm{T}}$. The $\mathrm{Z}+$ jets process is an important background for many standard model (SM) measurements and searches for physics beyond the SM. Measurements of differential cross sections for Z boson production in association with jets can be used to test models of initial-state radiation, and multiparton interactions (MPI). Monte Carlo (MC) models are used to simulate collision processes and play a crucial role in understanding background for the new physics searches. The description of MPI is an important part of these simulations. However, MPI can not be completely described by perturbative quantum chromodynamics (QCD), so this requires a phenomenological description involving parameters that must be tuned with the help of data.

The ATLAS, CMS, and LHCb experiments have reported various measurements of $\mathrm{Z}+$ jets processes at centre-of-mass energies of $7 \mathrm{TeV}$ [1-6], $8 \mathrm{TeV}[7-9]$, and $13 \mathrm{TeV}[10,11]$. $\mathrm{Z}$ boson production in association with jets is a process with a clean experimental signature and is well understood theoretically. In this paper, measurements of $\mathrm{Z}+$ jets events are performed to explore observables sensitive to the presence of MPI. A possible contribution could come from the simultaneous occurrence of two parton-parton interactions and is usually, when the involved scale is large, called double-parton scattering (DPS). This analysis explores new signatures sensitive to DPS in events with a $\mathrm{Z}$ boson and one or more jets, 

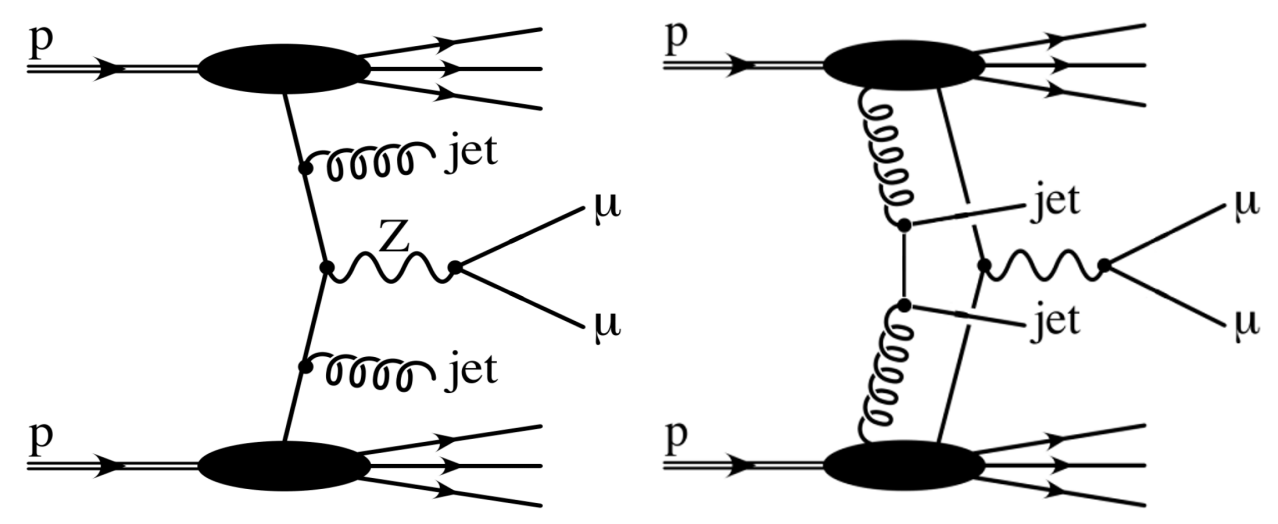

Figure 1. Typical diagrams for $\mathrm{Z}+2$ jet production in a single (left) and double-parton (right) scattering process.

and compares the data with MC simulations, using a variety of event generators simulating $\mathrm{Z}+$ jets processes with different MPI and hadronization models.

The ATLAS, ALICE, CMS, and LHCb Collaborations have previously reported measurements of DPS in various topologies, such as WW [12], W+jets [13, 14], 4 jets [15], J/ $\psi$ production [16-19], double charm production [20,21], but not in the Z+jets topology.

Figure 1 shows typical diagrams of single parton scattering (SPS) and DPS production of $\mathrm{Z}+2$ jet events. In SPS, the $\mathrm{Z}$ boson decaying into two muons and the two jets come from the same parton-parton interaction, whereas in the case of DPS, the Z boson and the two jets originate from two independent interactions.

Events are categorized as $\mathrm{Z}+\geq 1$ jet and $\mathrm{Z}+\geq 2$ jets topologies, and the corresponding integrated cross sections are measured in a fiducial region. For consistency with previous DPS measurements at $7 \mathrm{TeV}[13,14]$, jets are required to have a $p_{\mathrm{T}}$ threshold of $20 \mathrm{GeV}$. Decreasing the jet $p_{\mathrm{T}}$ threshold would increase the DPS contribution, but will also lead to a significant increase in the experimental uncertainties related to jet identification. With this threshold of $20 \mathrm{GeV}$, the DPS contribution is measured to be around $5.5 \%$ at $7 \mathrm{TeV}$ [14] and, as estimated from simulation, at $13 \mathrm{TeV}$ it is expected to increase by $20-30 \%$ because of the increased parton density. The differential cross sections are measured as functions of various observables based on the azimuthal ( $\phi$, in radians) separation and the $p_{\mathrm{T}}$ balance between the $\mathrm{Z}$ boson and jets, as well as the $p_{\mathrm{T}}$ balance between two jets in case of $\mathrm{Z}+$ $\geq 2$ jets events $[13,14,22]$. Since the two interactions in DPS are largely uncorrelated in the phase space studied, the shape of the distribution of these observables is expected to differ from that seen in SPS. Distributions of these observables normalized to the integrated cross section are measured because they have a lower systematic uncertainty due to the cancellation of the uncertainties correlated among bins. Previously measured distributions were used to extract parameters for DPS modelling in MC event generators [23, 24].

The measurement is performed using events where the $\mathrm{Z}$ boson decays into two oppositely charged muons. The pp collision data used in the analysis were collected in 2016 at $\sqrt{s}=13 \mathrm{TeV}$, corresponding to an integrated luminosity of $35.9 \mathrm{fb}^{-1}$. Tabulated results are provided in the HEPDATA [25]. 


\section{The CMS detector}

The central feature of the CMS apparatus is a superconducting solenoid of $6 \mathrm{~m}$ internal diameter, providing a magnetic field of $3.8 \mathrm{~T}$. Within the solenoid volume are a silicon pixel and strip tracker covering a pseudorapidity region of $|\eta|<2.5$, a lead tungstate crystal electromagnetic calorimeter (ECAL), and a brass and scintillator hadron calorimeter, each composed of a barrel and two endcap sections. Forward calorimeters, made of steel and quartz fibers, extend the $\eta$ coverage provided by the barrel and endcap detectors to $|\eta|<$ 5.0. Muons are measured in the range $|\eta|<2.4$, with detection planes made using three technologies: drift tubes, cathode strip chambers, and resistive plate chambers [26].

Events of interest are selected using a two-tiered trigger system. The first level, composed of custom hardware processors, uses information from the calorimeters and muon detectors to select events at a rate of about $100 \mathrm{kHz}$ within a latency of less than $4 \mu \mathrm{s}$ [27]. The second level, known as the high-level trigger, consists of a farm of processors running a version of the full event reconstruction software optimized for fast processing, and reduces the event rate to around $1 \mathrm{kHz}$ before data storage [28].

A more detailed description of the CMS detector, together with a definition of the coordinate system used and the relevant kinematic variables, is reported in ref. [29].

\section{Simulated event samples}

$\mathrm{MC}$ event generators are used to simulate the signal and background contributions. These MC samples are employed to optimize the event selection, to validate simulated event samples, to estimate some background sources, and to extract the unfolding response matrices used to correct for detector effects in the measured distributions. In addition, MC generators with different MPI and hadronization models are used to compare with the measured distributions and to evaluate systematic uncertainties related to the model dependence.

The Z+jets events are simulated with MADGRAPH5_aMC@NLO version 2.2.2 [30] (denoted MG5_aMC). The calculation includes matrix elements (MEs) computed at next-toleading-order (NLO) in perturbative QCD for the process $\mathrm{pp} \rightarrow \mathrm{Z}+N$ jets, $N=0,1$, or 2 . The sample is generated using the NNPDF 3.0 NLO parton distribution function (PDF) set [31]. An alternative simulation uses MG5_aMC to calculate leading-order (LO) MEs for $\mathrm{pp} \rightarrow \mathrm{Z}+N$ jets, $N=0, \ldots, 4$, and the NNPDF 3.0 LO PDF set.

The $\mathrm{Z}+$ jets process is also simulated with SHERPA v2.2 [32] with up to two additional parton emissions at NLO accuracy or up to four additional parton emissions at LO accuracy. The merging with the SHERPA parton shower is done via the MEPS@NLO prescription [33$35]$ using the five-flavour-number scheme, with a matching scale of $20 \mathrm{GeV}$. The NNPDF3.0 next-to-NLO (NNLO) set [31] and a dedicated set of tuned parton shower parameters [32] developed by the SHERPA authors are used.

Top quark-antiquark pair ( $(\bar{t})$ production, which forms the dominant background, is generated at NLO by MG5_aMC. The diboson (WW, WZ, ZZ) background processes are simulated at LO using PYTHIA8 v8.212 [36], and POWHEG 2.0 is used for the simulation of the single top quark processes ( $s$-channel, $t$-channel, and $t \mathrm{~W})$. The prediction for $\mathrm{t} \overline{\mathrm{t}}$ production is normalized to NNLO in QCD including resummation of next-to-next-to- 
leading logarithmic soft-gluon terms with Top ++2.0 [37-43]. Diboson and single top quark background predictions are normalized to NLO [44] and NNLO [45] cross sections, respectively.

All samples, except those based on SHERPA, use PYTHIA 8 to model the initial- and finalstate parton showers and hadronization with the CUETP8M1 [24] or CUETP8M2T4 [46] tune. The CUETP8M1 tune includes the NNPDF 2.3 [47] LO PDF set with the strong coupling $\alpha_{\mathrm{S}}\left(m_{\mathrm{Z}}\right)$ set to 0.1365 for space- and time-like shower simulation. The CUETP8M2T4 tune is based on the CUETP8M1 tune, which includes the NNPDF30_lo_as_0130 PDF set, but uses a lower value of $\alpha_{\mathrm{S}}=0.1108$ for the initial-state radiation component of the parton shower. Matching between the ME generators and the parton shower is done using the $k_{\mathrm{T}}$-MLM scheme $[48,49]$ with the matching scale set at $19 \mathrm{GeV}$ for the LO MG5_aMC sample, and the FxFx [50] scheme with the matching scale set to $30 \mathrm{GeV}$ for the NLO MG5_aMC events.

Generated events are processed through a full GEANT4-based [51] CMS detector simulation and trigger emulation. The simulated samples include the effects of multiple interactions in each bunch crossing, referred to as pileup. The simulated events are reconstructed with the same algorithm used for the data.

In addition to the $\mathrm{Z}+$ jets MG5_aMC samples with the CUETP8M1 tune described above, the measurements are compared with simulations using different PYTHIA 8 tunes, such as CP5 [23], CP5 without MPI, and CDPSTP8S1-Wj [24]. The CP5 tune uses NNPDF3.1 PDF set at NNLO, with $\alpha_{\mathrm{S}}$ values of 0.118 , and running according to NLO evolution. The CP5 tune is chosen since it is the standard tune obtained by fitting a large number of 1.96, 7, and $13 \mathrm{TeV}$ measurements sensitive to soft and semihard multipartonic interactions [23]. The $\mathrm{W}+$ dijet DPS tune, CDPSTP8S1-Wj is derived from the parameters of PYTHIA 8 tune $4 \mathrm{C}$, with a variation of the impact parameter dependence, i.e. matter overlap function, which is the convolution of the matter distributions of the two incoming hadrons. The DPS simulation in MC is usually quantified in terms of a parameter known

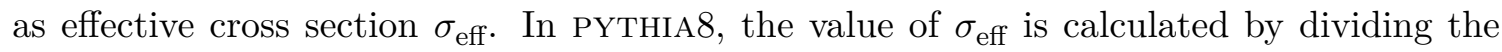
nondiffractive (ND) cross section by the so-called "enhancement factor", which depends on the parameters of the overlap matter distribution function and the limiting value of $p_{\mathrm{T}}$ at a reference energy [52]. For central collisions, the enhancement factor tends to be large, translating to a lower value of $\sigma_{\text {eff }}$ and larger DPS contribution. For peripheral interactions, enhancement factors are small, giving large values of $\sigma_{\text {eff }}$ and a small DPS contribution.

The $\mathrm{Z}+$ jets events calculated at NLO with MG5_aMC are also interfaced with HERWIG7, applying the $\mathrm{CH} 3$ tune for the underlying event description, hadronization, and showering [53-56]. The tune is derived by fitting measurements from pp collision data collected by the CMS experiment at $\sqrt{s}=0.9,7$, and $13 \mathrm{TeV}$. The CH3 tune makes use of the NNPDF3.1 LO PDF set with $\alpha_{\mathrm{S}}=0.130$ for the simulation of MPI, but the NNLO PDF set with $\alpha_{\mathrm{S}}=0.118$ for all other components. The cross sections of $\mathrm{Z}+$ jets events simulated with different $\mathrm{MC}$ event generators and configurations are normalized to NNLO calculations with FEWZ v3.1 [57]. Table 1 summarizes all the event generators, PDF sets, and tunes used to produce both signals (including alternative samples), and the background processes. 


\begin{tabular}{|c|c|c|c|}
\hline Process name & Event generator (k-factor) & Tune & PDF set \\
\hline $\mathrm{Z}+$ jets & MG5_aMC (NLO) + PYTHIA8 (1.07) & CUETP8M1 & $\begin{array}{l}\text { NNPDF } 3.0 \text { NLO for matrix element } \\
\text { NNPDF 2.3 LO for tune }\end{array}$ \\
\hline $\mathrm{Z}+$ jets & MG5_aMC (LO) + PYTHIA8 (1.24) & CUETP8M1 & $\begin{array}{l}\text { NNPDF } 3.0 \text { LO for matrix element } \\
\text { NNPDF 2.3 LO for tune }\end{array}$ \\
\hline $\mathrm{Z}+$ jets & SHERPA (NLO) (0.97) & - & NNPDF 3.0 NNLO \\
\hline $\mathrm{Z}+$ jets & MG5_aMC (NLO) + PYTHIA8 (1.07) & CP5 & $\begin{array}{l}\text { NNPDF } 3.0 \text { NLO for matrix element } \\
\text { NNPDF } 3.1 \text { NNLO for tune }\end{array}$ \\
\hline $\mathrm{Z}+$ jets & MG5_aMC (NLO) + PYTHIA8 MPI-OFF (1.07) & CP5 & $\begin{array}{l}\text { NNPDF } 3.0 \text { NLO for matrix element } \\
\text { NNPDF } 3.1 \text { NNLO for tune }\end{array}$ \\
\hline $\mathrm{Z}+$ jets & MG5_aMC (NLO) + PYTHIA8 (1.09) & CDPSTP8S1-Wj & $\begin{array}{l}\text { NNPDF } 3.0 \text { NLO for matrix element } \\
\text { CTEQ6L1 LO for tune }\end{array}$ \\
\hline $\mathrm{Z}+$ jets & MG5_aMC (NLO) + HERWIG7 (1.02) & $\mathrm{CH} 3$ & $\begin{array}{l}\text { NNPDF } 3.0 \text { NLO for matrix element } \\
\text { NNPDF } 3.1 \mathrm{LO} / \mathrm{NNLO} \text { for tune }\end{array}$ \\
\hline$t \bar{t}$ & MG5_aMC (NLO) + PYTHIA8 (一) & CUETP8M2T4 & $\begin{array}{l}\text { NNPDF } 3.0 \text { NLO for matrix element } \\
\text { NNPDF30_lo_as_0130 for tune }\end{array}$ \\
\hline Diboson & PYTHIA8 (LO) $(-)$ & - & NNPDF $2.3 \mathrm{LO}$ \\
\hline Single top & POWHEG (NLO) + PYTHIA8 (一) & CUETP8M1 & $\begin{array}{l}\text { NNPDF } 3.0 \text { NLO for matrix element } \\
\text { NNPDF 2.3 LO for tune }\end{array}$ \\
\hline
\end{tabular}

Table 1. Summary of event generators with their k-factors (ratio of NNLO to NLO/LO cross section), PDF sets, and tunes used to produce both the signal and background event samples.

\section{Event selection}

The particle flow (PF) algorithm [58] is used to reconstruct and identify individual particle candidates in an event, with an optimized combination of information from the various elements of the CMS detector. Energy deposits are measured in the calorimeters and charged particles are identified in the central tracking and muon systems.

Events are selected with a single-muon trigger requiring at least one isolated muon candidate with $p_{\mathrm{T}}>24 \mathrm{GeV}$. The primary vertex is the vertex candidate with the largest value of summed physics-object $p_{\mathrm{T}}^{2}$. The physics objects are the track-only jets, clustered using the jet finding anti- $k_{\mathrm{T}}$ algorithm $[59,60]$ with the tracks assigned to candidate vertices as inputs, and the associated missing $p_{\mathrm{T}}$, which is the negative vector $p_{\mathrm{T}}$ sum of all physics objects. Events are required to have at least two oppositely charged muons with $p_{\mathrm{T}}>$ $27 \mathrm{GeV}$ and $|\eta|<2.4$. These muons are reconstructed by combining information from the inner tracker and the muon detector subsystems. The muon candidates are required to satisfy identification criteria based on the number of hits in each detector, the quality of the muon-track fit, and the consistency with the primary vertex, which is imposed by requiring the longitudinal and transverse impact parameters are less than 0.5 and $0.2 \mathrm{~cm}$, respectively. The efficiency to reconstruct and identify the muons is greater than $96 \%$. Matching muon candidates to tracks measured in the silicon tracker results in a relative $p_{\mathrm{T}}$ resolution for muons with $20<p_{\mathrm{T}}<100 \mathrm{GeV}$ of $1 \%$ in the barrel and $3 \%$ in the endcaps.

To suppress the multijet background, muons are required to be isolated. The relative isolation variable, $\mathrm{I}_{\mathrm{rel}}$, for muons is defined as:

$$
\mathrm{I}_{\mathrm{rel}}=\frac{\left[\sum p_{\mathrm{T}}^{\text {charged }}+\max \left(0 ., \sum E_{\mathrm{T}}^{\text {neutral }}+\sum E_{\mathrm{T}}^{\gamma}-0.5 \sum p_{\mathrm{T}}^{\mathrm{PU}}\right)\right]}{p_{\mathrm{T}}^{\mu}} .
$$


Here $\sum E_{\mathrm{T}}^{\text {neutral }}$ and $\sum E_{\mathrm{T}}^{\gamma}$ are the transverse energy sums of neutral hadrons and photons, respectively, within a cone of radius $\Delta R=\sqrt{(\Delta \eta)^{2}+(\Delta \phi)^{2}}=0.4$ around the muon track. The quantity $\sum p_{\mathrm{T}}^{\text {charged }}$ represents the $p_{\mathrm{T}}$ sum of the charged hadrons in the same cone around the muon associated with the selected vertex. Finally, $\sum p_{\mathrm{T}}^{\mathrm{PU}}$ is the $p_{\mathrm{T}}$ sum of the charged hadrons in the same cone around the muon not associated with the selected vertex. A muon is considered isolated if $\mathrm{I}_{\text {rel }}<0.2$. There are small residual differences in the trigger, identification and isolation efficiencies between data and simulation, which are measured using the "tag-and-probe" method [61], and included by applying scale factors to simulated events [26].

To select $\mathrm{Z}$ boson candidate events, the invariant mass of the two oppositely charged muons with highest $p_{\mathrm{T}}$ is required to be close to the $\mathrm{Z}$ boson mass, $70<m_{\mu^{+} \mu^{-}}<110 \mathrm{GeV}$. The $\mathrm{Z}$ boson candidate is required to be accompanied by at least one jet with $p_{\mathrm{T}}>20 \mathrm{GeV}$ and $|\eta|<2$.4. The overlap between the muons from the $\mathrm{Z}$ boson decay and the jets is removed by requiring a minimum $\Delta R$ distance of 0.4 between them.

Jets are clustered from PF candidates using the infrared- and collinear-safe anti- $k_{\mathrm{T}}$ algorithm [59] with a distance parameter of 0.4, as implemented in the FASTJET package [60]. The jet momentum is determined as the vectorial sum of all particle momenta in the jet, and is found from simulation to be, on average, within 5 to $10 \%$ of the true momentum over the entire $p_{\mathrm{T}}$ spectrum and detector acceptance. Pileup can contribute additional tracks and calorimetric energy depositions, increasing the apparent jet momentum. To mitigate the effects of pileup, tracks identified as originating from pileup vertices are discarded, and a factor [62] is applied to correct for the remaining contributions. Jet energy corrections are derived from simulation studies so that the average measured response of jets becomes identical to that of particle-level jets. In situ measurements of the momentum balance in dijet, multijet, photon + jet, and $\mathrm{Z}+$ jets events with the $\mathrm{Z}$ boson decaying leptonically are used to correct residual differences between the jet energy scales in data and simulation $[63,64]$. The jet energy in simulation is spread to match the resolution observed in data. The jet energy resolution (JER) in data amounts typically to $15-20 \%$ at jet energy of $30 \mathrm{GeV}, 10 \%$ at $100 \mathrm{GeV}$, and $5 \%$ at $1 \mathrm{TeV}$. Additional selection criteria are applied to remove jets potentially dominated by anomalous contributions from various subdetector components or reconstruction failures [65]. Jets identified as being likely to originate from pileup $[66,67]$ are also removed by using a pileup jet ID discriminator for jets with $20<p_{\mathrm{T}} \leq 50 \mathrm{GeV}$. No pileup jet ID is applied to jets with $p_{\mathrm{T}} \geq 50 \mathrm{GeV}$, since here the pileup event contribution is negligible [67].

The selected events correspond to a sample of $Z+$ jets events with a background of $2-$ $5 \%$, which is subtracted from the data before the unfolding. The dominant contribution is the production of $t \bar{t}$ pairs. The simulation of $t \bar{t}$ production is validated with data in $a t \bar{t} \rightarrow$ $\mathrm{e} \mu+\mathrm{X}$ control region. The $\mathrm{t} \overline{\mathrm{t}}$ control region is constructed using data events by requiring an oppositely charged electron-muon pair with at least one jet. The energy of electrons is determined from a combination of the electron momentum at the primary interaction vertex as determined by the tracker, the energy of the corresponding ECAL cluster, and the energy sum of all bremsstrahlung photons spatially compatible with originating from the 
electron track [68]. The longitudinal and transverse impact parameters for barrel (endcap) are required to be less than $0.10(0.20)$ and $0.05(0.10) \mathrm{cm}$, respectively. Electrons are required to have $p_{\mathrm{T}}>27 \mathrm{GeV}$ and $|\eta|<2.4$. The selection criteria for muons and jets are the same as discussed above. The invariant mass of the oppositely charged e $\mu$ pair is required to lie within the range of $70<m_{\mathrm{e} \mu}<110 \mathrm{GeV}$. The difference in the data to simulation comparison in this control region is included as a part of the systematic uncertainty.

\section{$5 \quad$ Observables and the unfolding}

Events are categorized in the $\mathrm{Z}+\geq 1$ jet and $\mathrm{Z}+\geq 2$ jets subsets, and the differential cross sections are measured as function of the following observables:

1. $\mathrm{Z}+\geq 1$ jet events

- Azimuthal angle between the $\mathrm{Z}$ boson and the highest $p_{\mathrm{T}}$ (leading) jet: $\Delta \phi\left(\mathrm{Z}, j_{1}\right)$. For SPS events, the $\mathrm{Z}$ boson and the leading jet will balance each other, hence this observable will peak around $\pi$, whereas for the DPS process the distribution is expected to be flat because of the absence of a correlation between the $\mathrm{Z}$ boson and the leading jet produced from the two different scatterings.

- Relative $p_{\mathrm{T}}$ imbalance between the $\mathrm{Z}$ boson and leading jet is

$$
\Delta_{\text {rel }} p_{\mathrm{T}}\left(\mathrm{Z}, j_{1}\right)=\frac{\left|\overrightarrow{p_{\mathrm{T}}}(\mathrm{Z})+\overrightarrow{p_{\mathrm{T}}}\left(j_{1}\right)\right|}{\left|\overrightarrow{p_{\mathrm{T}}}(\mathrm{Z})\right|+\left|\overrightarrow{p_{\mathrm{T}}}\left(j_{1}\right)\right|} .
$$

This observable is expected to be close to zero for SPS events, whereas in the case of DPS this observable will have higher values.

2. $\mathrm{Z}+\geq 2$ jets events

- Azimuthal angle between the $\mathrm{Z}$ boson and dijet system: $\Delta \phi(\mathrm{Z}$, dijet). Here, dijet means the resulting three-momentum of the leading and subleading jets. For SPS events, the dijet system $p_{\mathrm{T}}$ will balance the $\mathrm{Z}$ boson $p_{\mathrm{T}}$, therefore, this observable will peak around $\pi$. In the case of DPS production, the distribution is expected to be flat since the $\mathrm{Z}$ boson and the two jets are originating from two independent scatterings.

- Relative $p_{\mathrm{T}}$ imbalance between the $\mathrm{Z}$ boson and dijet system is

$$
\Delta_{\text {rel }} p_{\mathrm{T}}(\mathrm{Z}, \text { dijet })=\frac{\mid \overrightarrow{p_{\mathrm{T}}}(\mathrm{Z})+\overrightarrow{p_{\mathrm{T}}}(\text { dijet }) \mid}{\left|\overrightarrow{p_{\mathrm{T}}}(\mathrm{Z})\right|+\mid \overrightarrow{p_{\mathrm{T}}}(\text { dijet }) \mid} .
$$

For SPS events this observable is expected to be 0, whereas for DPS events this observable will have higher values.

- Relative $p_{\mathrm{T}}$ imbalance between the leading $\left(j_{1}\right)$ and subleading $\left(j_{2}\right)$ jets is

$$
\Delta_{\text {rel }} p_{\mathrm{T}}\left(j_{1}, j_{2}\right)=\frac{\left|\overrightarrow{p_{\mathrm{T}}}\left(j_{1}\right)+\overrightarrow{p_{\mathrm{T}}}\left(j_{2}\right)\right|}{\left|\overrightarrow{p_{\mathrm{T}}}\left(j_{1}\right)\right|+\left|\overrightarrow{p_{\mathrm{T}}}\left(j_{2}\right)\right|} .
$$




\begin{tabular}{|lc|}
\hline Object & Selections \\
\hline Muons (dressed) & $p_{\mathrm{T}}>27 \mathrm{GeV},|\eta|<2.4$ \\
Z boson & $70<m_{\mu^{+}{ }_{\mu}}<110 \mathrm{GeV}$ \\
At least 1 jet & $p_{\mathrm{T}}>20 \mathrm{GeV},|\eta|<2.4$ \\
\hline
\end{tabular}

Table 2. Fiducial selections at particle level.

For DPS events, the two jets are expected to balance each other, therefore this observable will be around 0. For SPS events, the two jets are correlated with the $\mathrm{Z}$ boson and not expected to balance each other.

The reconstructed distributions are corrected for the event selection efficiency and detector resolution using an unfolding technique that employs a response matrix to map the reconstructed observables onto the particle-level values. The unfolding is done with 20 detector-level and 10 particle-level $\Delta \phi$ or $\Delta_{\text {rel }} p_{\mathrm{T}}$ bins. The unfolding is performed using the TUNFOLD package [69], which is based on a least squares fit with a possible Tikhonov regularization term [70]. Since the effect of regularization is minimal on the reported observables, the unfolding is performed without regularization.

The $\mathrm{Z}+$ jets events simulated with MG5_aMC + PYTHIA8 with tune CUETP8M1 are used to construct the response matrix. At particle level, events are required to have at least two oppositely charged muons with $p_{\mathrm{T}}>27 \mathrm{GeV}$ and $|\eta|<2.4$. The particle-level definition of a muon corresponds to a generator-level muon coming from the $\mathrm{Z}$ boson decay, "dressed" by adding the momenta of all photons within $\Delta R<0.1$ around both muon directions to account for the FSR effects [71]. The particle-level jets with $p_{\mathrm{T}}>20 \mathrm{GeV}$ and $|\eta|<2.4$ are formed from stable particles $(c \tau>1 \mathrm{~cm})$, except neutrinos, using the same anti- $k_{\mathrm{T}}$ jet algorithm as for reconstructed jets. A possible overlap between particle-level jets and a pair of muons from the $\mathrm{Z}$ boson decay is removed by requiring a minimum distance of 0.4 between them. The distributions are unfolded to the particle level in the fiducial region defined in table 2 .

In simulation, the reconstructed jets and a pair of muons are spatially matched to the corresponding particle-level objects by requiring that they are within $\Delta R$ of 0.4 from one another. Events that have reconstructed objects without matched particle-level objects are included in the background category and are excluded from the sample. This contribution includes the events where the selected jets originate from pileup. The contribution of background with no jet at particle level, but at least one jet at the reconstructed level is about $4.1 \%$. The contribution of background with no jet (1 jet) at particle level and at least 2 jets at the reconstruction level is around $1.1(4.8) \%$. The simulation of pileup jets is validated in a control region enriched in pileup jets, obtained by inverting the criteria used to reject the pileup jets. The simulation describes the data well, within the uncertainties, in the pileup-enriched control region, validating the simulation of background events from pileup.

Events that have particle-level objects in the fiducial volume, but no matching reconstructed objects, are accounted for with acceptance and efficiency corrections. In addition, 
there may be events in which the particle-level jet passing the fiducial selection does not lead to a reconstructed level jet that passes the fiducial selection. Events of this type are considered as background at the reconstruction level and are not considered. However, at the generator level, these are genuine signal events missed because of the detector and reconstruction inefficiencies. These missed events are accounted for via a signal acceptance correction.

Finally, the unfolded distributions are scaled with the inverse of the integrated luminosity to obtain the differential cross section.

\section{$6 \quad$ Systematic uncertainties}

The measurements have various sources of systematic uncertainties.

- Jet energy resolution and scale: the effect of the uncertainty in jet energy scale (JES) or JER $[63,64]$ is evaluated by varying the JES or JER within the associated uncertainty and performing the unfolding procedure with the modified distribution. The variations of JES corrections within their uncertainties change the differential cross sections by $2-8 \%$, whereas the area-normalized distributions are affected by up to $4 \%$. The variations of JER corrections within their uncertainty change the differential cross section distributions by $1-7 \%$ and area-normalized distributions up to $5 \%$.

- Pileup jet identification: simulated events are corrected for the differences in the jet identification efficiency between data and simulated events. The uncertainties in these corrections affect the measurements by up to 1-2\% for differential cross section distributions and less than $0.5 \%$ for area-normalized distributions.

- Closure uncertainty: the effect of model dependence is evaluated by comparing unfolded results obtained using response matrices constructed with the MG5_aMC + PYTHIA8 with tune CUETP8M1 and SHERPA generators having different ME and parton showering, as discussed in section 3. The calculated uncertainty is then symmetrized.

The effect of scale uncertainties is estimated using a set of generator weights that correspond to variations of renormalization $\left(\mu_{F}\right)$ and factorization $\left(\mu_{R}\right)$ scales up and down by factors of 2 from their nominal values, excluding the pair of extreme variations. The unfolded distributions are obtained for all such combinations and their envelope is quoted as the uncertainty.

The uncertainty in the PDFs is estimated using the 100 replicas of the NNPDF 3.0 PDF set [72]. The unfolded distributions are reproduced using the weights of the replicas and a standard deviation is computed on a bin-by-bin basis [72].

These sources, added in quadrature, affect the differential cross section by $1-5 \%$ and area-normalized distributions up to $1-4 \%$ in the case of $\mathrm{Z}+\geq 1$ jet events, whereas the effect is within $9 \%$ for the differential cross section and up to $7 \%$ for the areanormalized distribution in the case of $\mathrm{Z}+\geq 2$ jet events. 
- Integrated luminosity: it is determined with a 2.5\% [73] uncertainty for differential cross section distributions, but completely cancels in the area normalized distributions.

- Pileup weighting: the distribution of the mean number of interactions per bunch crossing of the simulated samples is weighted to match that of the data. The uncertainty related to pileup weighting is estimated by varying the total inelastic cross section by $\pm 4.6 \%$ [74]. The effect is negligible for both the differential and areanormalized distributions. After the pileup weighting, the vertex multiplicity in simulation shows overall good agreement with data, but there is an overestimation of around $40 \%$ for high vertex multiplicities. To investigate the effect of this residual discrepancy, the simulated events are additionally corrected to reproduce the vertex multiplicity distributions observed in data. The data are unfolded with the weighted response matrix, and the results are compared with the unfolded results without weighting. These sources, added in quadrature, affect the differential cross section by $1.0-1.5 \%$ and area-normalized distributions up to $1 \%$.

- Muon selection: the systematic uncertainty related to various muon selection criteria such as muon identification, isolation and trigger scale factors is less than $1 \%$ for both differential cross section and area-normalized distributions.

The effect of the muon momentum corrections on the measurement is very small $(<0.1 \%)$, therefore no additional systematic uncertainty is assigned.

- Background modelling: there is a small contribution from t $\bar{t}$ events, whereas the contribution from other processes such as dibosons, $\mathrm{W}+$ jets, and QCD multijet production is smaller than $1 \%$. To calculate the systematic uncertainty related to the simulated background contribution, the cross sections of the background samples are varied by their uncertainties. The systematic uncertainty related to the t $\overline{\mathrm{t}}$ process is obtained from the differences between data and simulation in a $t \bar{t} \rightarrow \mathrm{e} \mu+\mathrm{X}$ control region. The variation of the background contribution within the uncertainties affects the differential cross section less than $0.2 \%$ for $\mathrm{Z}+\geq 1$ jet and less than $0.6 \%$ for $\mathrm{Z}+$ $\geq 2$ jets events. The effect of this variation on the area-normalized distributions is less than $0.2 \%$.

Tables 3 and 4 summarize the effect of various systematic uncertainties for the differential cross section and the normalized distributions. These systematic uncertainties are considered uncorrelated and are added in quadrature.

\section{$7 \quad$ Results}

The production cross sections in the fiducial region defined in table 2 are measured to be $158.5 \pm 0.3$ (stat) \pm 7.0 (syst) \pm 1.2 (theo) \pm 4.0 (lumi) pb for $\mathrm{Z}+\geq 1$ jet events and $44.8 \pm 0.4$ (stat) \pm 3.7 (syst) \pm 0.5 (theo) \pm 1.1 (lumi) pb for $\mathrm{Z}+\geq 2$ jet events. The measured cross sections are described, within the uncertainties, by different simulations, except for the MG5_aMC + PYTHIA8 with CP5 tune MPIOFF and the DPS-specific CDPSTP8S1WJ tune. The cross section of the DPS-specific tune is predicted to be $10 \%$ higher than 


\begin{tabular}{|lccccc|}
\hline Observable/Uncertainty & $\Delta \phi\left(\mathrm{Z}, j_{1}\right)$ & $\Delta_{\text {rel }} p_{\mathrm{T}}\left(\mathrm{Z}, j_{1}\right)$ & $\Delta \phi(\mathrm{Z}$, dijet $)$ & $\Delta_{\text {rel }} p_{\mathrm{T}}(\mathrm{Z}$, dijet $)$ & $\Delta_{\text {rel }} p_{\mathrm{T}}\left(j_{1}, j_{2}\right)$ \\
\hline JES & $2.7-7.5 \%$ & $2.4-7.4 \%$ & $4.9-7.9 \%$ & $4.5-8.4 \%$ & $4.4-7.3 \%$ \\
JER & $0.9-6.6 \%$ & $1.4-5.8 \%$ & $1.2-7.2 \%$ & $2.1-5.1 \%$ & $1.1-4.2 \%$ \\
Pileup jet identification & $1.3-1.7 \%$ & $0.9-1.6 \%$ & $1.7-2.1 \%$ & $1.6-2.1 \%$ & $1.7-2.3 \%$ \\
Integrated luminosity & $2.5 \%$ & $2.5 \%$ & $2.5 \%$ & $2.5 \%$ & $2.5 \%$ \\
Pileup modelling & $0.1-0.7 \%$ & $0.2-1.0 \%$ & $0.2-1.4 \%$ & $0.4-1.4 \%$ & $0.8-1.4 \%$ \\
Closure uncertainty & $0.6-4.0 \%$ & $0.8-5.1 \%$ & $2.7-6.1 \%$ & $2.2-8.7 \%$ & $2.2-8.7 \%$ \\
Muon selection & $<1.0 \%$ & $<1.0 \%$ & $<1.0 \%$ & $<1.0 \%$ & $<1.0 \%$ \\
Background modelling & $<0.2 \%$ & $<0.2 \%$ & $<0.6 \%$ & $<0.6 \%$ & $<0.4 \%$ \\
Total & $4-11 \%$ & $4-10 \%$ & $8-14 \%$ & $8-14 \%$ & $7-11 \%$ \\
\hline
\end{tabular}

Table 3. Uncertainty sources and their effect on the differential cross section distributions.

\begin{tabular}{|lccccc|}
\hline Observable/Uncertainty & $\Delta \phi\left(\mathrm{Z}, j_{1}\right)$ & $\Delta_{\text {rel }} p_{\mathrm{T}}\left(\mathrm{Z}, j_{1}\right)$ & $\Delta \phi(\mathrm{Z}$, dijet $)$ & $\Delta_{\text {rel }} p_{\mathrm{T}}(\mathrm{Z}$, dijet $)$ & $\Delta_{\text {rel }} p_{\mathrm{T}}\left(j_{1}, j_{2}\right)$ \\
\hline JES & $0.1-3.8 \%$ & $0.7-3.7 \%$ & $0.6-4.0 \%$ & $0.3-2.6 \%$ & $0.3-1.5 \%$ \\
JER & $0.3-4.6 \%$ & $0.4-4.4 \%$ & $1.3-4.4 \%$ & $0.2-4.8 \%$ & $0.2-1.7 \%$ \\
Pileup jet identification & $0.1-0.2 \%$ & $0.1-0.2 \%$ & $0.1-0.2 \%$ & $0.1-0.2 \%$ & $0.1-0.4 \%$ \\
Pileup modelling & $0.1-0.5 \%$ & $0.1-0.5 \%$ & $0.1-1 \%$ & $0.1-0.8 \%$ & $0.2-0.4 \%$ \\
Closure uncertainty & $0.8-2.5 \%$ & $0.9-3.6 \%$ & $0.3-5.0 \%$ & $0.4-6.7 \%$ & $0.5-3.7 \%$ \\
Muon selection & $<1.0 \%$ & $<1.0 \%$ & $<1.0 \%$ & $<1.0 \%$ & $<1.0 \%$ \\
Background modelling & $<0.1 \%$ & $<0.1 \%$ & $<0.2 \%$ & $<0.2 \%$ & $<0.2 \%$ \\
Total & $1-6 \%$ & $1-6 \%$ & $2-7 \%$ & $1-7 \%$ & $1-4 \%$ \\
\hline
\end{tabular}

Table 4. Uncertainty sources and their effect on the area-normalized distributions.

the measured cross section. The cross section of CP5 tune without MPI underestimates the measured cross section up to $10 \%$ for $\mathrm{Z}+\geq 1$ jet events and up to $16 \%$ for $\mathrm{Z}+\geq 2$ jet events. Table 5 summarizes the measured and predicted cross sections for the $\mathrm{Z}+\geq 1$ jet and $\mathrm{Z}+\geq 2$ jet processes.

For $\mathrm{Z}+\geq 1$ jet events, figure 2 (3) shows the differential cross section measurements (left) and the area-normalized distributions (right) as a function of $\Delta \phi\left(\mathrm{Z}, j_{1}\right)$ $\left(\Delta_{\text {rel }} p_{\mathrm{T}}\left(\mathrm{Z}, j_{1}\right)\right)$, respectively. Different MC event generators (except for the MG5_aMC + PYTHIA8 with the DPS-specific tune CDPSTP8S1-WJ) describe, within the uncertainties, the overall differential cross section as a function of $\Delta \phi$ and $\Delta_{\text {rel }} p_{\mathrm{T}}$, apart from a few discrepancies in some specific regions of these observables. MG5_aMC + PYTHIA8 generator prediction with the DPS-specific tune CDPSTP8S1-WJ overestimates the cross section up to 10-20\%, but correctly describe the shapes of the $\Delta \phi$ and $\Delta_{\text {rel }} p_{\mathrm{T}}$ distributions. The MG5_aMC + PYTHIA8 prediction (with CP5 tune) overestimates (up to 20\%) the measurement in the lower- $\Delta_{\text {rel }} p_{\mathrm{T}}$, where SPS is expected to be dominant. The prediction of MG5_aMC + HERWIG7 describes, within the uncertainties, the shape of the $\Delta_{\text {rel }} p_{\mathrm{T}}$ distribution, but deviates up to $15-20 \%$ in the lower- $\Delta \phi$ region where DPS is expected to contribute more. 


\begin{tabular}{|c|c|c|c|}
\hline \multicolumn{2}{|l|}{ Cross section $(\mathrm{pb})$} & $\mathrm{Z}+\geq 1$ Jets & $\mathrm{Z}+\geq 2$ Jets \\
\hline \multicolumn{2}{|l|}{ Measured in data } & $\begin{array}{l}158.5 \pm 0.3 \text { (stat) } \\
\pm 7.0 \text { (syst) } \\
\pm 1.2 \text { (theo) } \\
\pm 4.0 \text { (lumi) pb }\end{array}$ & $\begin{array}{l}44.8 \pm 0.4 \text { (stat) } \\
\pm 3.7 \text { (syst) } \\
\pm 0.5 \text { (theo) } \\
\pm 1.1 \text { (lumi) pb }\end{array}$ \\
\hline \multicolumn{4}{|l|}{ Predicted by MC } \\
\hline \multirow{4}{*}{ MG5_aMC (NLO) } & PYTHIA8, CP5 tune & $167.4 \pm 9.7$ & $47.0 \pm 3.9$ \\
\hline & PYTHIA8, CP5 tune MPIOFF & $143.8 \pm 0.3$ & $37.7 \pm 0.2$ \\
\hline & PYThia8, CDPSTP8S1-WJ tune & $178.4 \pm 0.3$ & $50.5 \pm 0.2$ \\
\hline & HERWIG7, CH3 tune & $158.3 \pm 1.1$ & $44.4 \pm 0.6$ \\
\hline \multirow{2}{*}{\multicolumn{2}{|c|}{$\begin{array}{l}\text { MG5_aMC (LO) + PYTHIA8, CP5 tune } \\
\text { SHERPA (NLO+LO) }\end{array}$}} & $161.2 \pm 0.1$ & $45.3 \pm 0.1$ \\
\hline & & $149.8 \pm 0.2$ & $41.6 \pm 0.1$ \\
\hline
\end{tabular}

Table 5. Measured and predicted cross section for $\mathrm{Z}+\geq 1$ jet and $\mathrm{Z}+\geq 2$ jet production. Simulations are normalized to NNLO calculations (from FEWZ) and reported cross section values are extracted by applying fiducial selections. Predicted cross sections include statistical and theoretical uncertainties added in quadrature for MG5_aMC + PYTHIA8 with CP5 tune, whereas the other predicted cross sections are reported with only statistical uncertainties.

The Z+jets calculation of MG5_aMC + PYTHIA8 without MPI does not describe the measurement and is lower than the measurement by up to $50 \%$ in both the lower $\Delta \phi$ and higher $\Delta_{\text {rel }} p_{\mathrm{T}}$ regions, where the MPI contribution is expected to be the largest.

For $\mathrm{Z}+\geq 2$ jet events figure 4 (5) shows the differential cross section measurements (left) and the area-normalized distributions (right) as a function of $\Delta \phi(\mathrm{Z}$, dijet) $\left(\Delta_{\text {rel }} p_{\mathrm{T}}(\mathrm{Z}\right.$, dijet $\left.)\right)$, respectively. The differential cross section, as a function of $\Delta \phi$, is reasonably well described by the different predictions. The predictions of MG5_aMC + PYTHIA8 (with the CDPSTP8S1-WJ tune) and SHERPA best describe the shape of the measured distributions, whereas the MG5_aMC + PYTHIA8 (with CP5 tune) prediction deviates by up to $15 \%$ in the lower- $\Delta \phi$ region, where DPS production of $\mathrm{Z}+$ jets is expected to contribute more. The shape of the $\Delta_{\text {rel }} p_{\mathrm{T}}$ distribution is best described by the predictions of MG5_aMC + PYTHIA8 (with the CDPSTP8S1-WJ tune) and SHERPA. The MG5_aMC + PYTHIA 8 (with CP5 tune) prediction overestimates, up to $20 \%$, in the lower- $\Delta_{\text {rel }} p_{\mathrm{T}}$ region.

The differential cross section as a function of $\Delta_{\text {rel }} p_{\mathrm{T}}$ between two jets, as shown in figure 6 , is well described by the different predictions except for MG5_aMC + PYTHIA8 (with the CDPSTP8S1-WJ tune), which overestimates the differential cross section measurements up to $15 \%$. The shape of the $\Delta_{\text {rel }} p_{\mathrm{T}}$ distribution is described well by the predictions presented, except some deviations shown by MG5_aMC + HERWIG7 mainly in the higher region of the distribution. If MPI is not included in the simulation, predictions underestimate the differential cross section and fail to describe the shape of all the observables for $\mathrm{Z}+\geq 2$ jet events with deviations up to $50 \%$.

The model parameters for different MPI and hadronization models are mostly derived by fitting minimum bias and low- $p_{\mathrm{T}}(\lesssim 3 \mathrm{GeV})$ MPI measurements. From the above data, it is clear that our measurements can test the accuracy of various predictions and the 

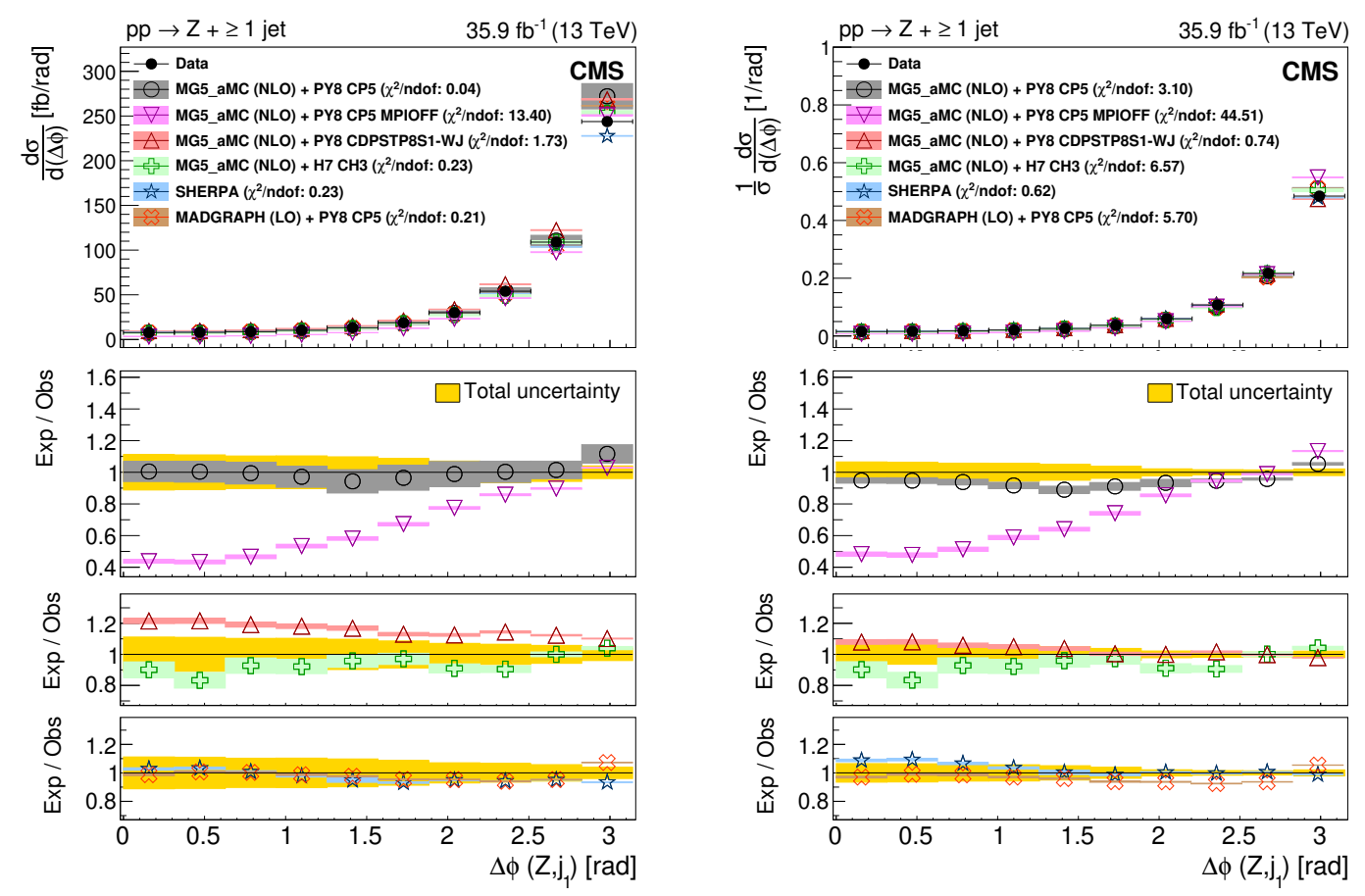

Figure 2. Differential cross sections (left) and area-normalized distributions (right) as functions of $\Delta \phi$ between the $\mathrm{Z}$ boson and the leading jet for $\mathrm{Z}+\geq 1$ jet events. The uncertainties in the predictions are shown as coloured bands around the theoretical predictions including statistical, PDF, scale, and tune uncertainties for the NLO MG5_aMC + PYTHIA8 (with CP5 tune) and the statistical uncertainty only for the LO MG5_aMC + PYTHIA8 (with CP5 tune), NLO MG5_aMC + PYTHIA8 (with CDPSTP8S1-WJ tune, CP5 tune with MPI-OFF), NLO MG5_aMC + HERWIG7 (with tune CH3), and SHERPA predictions. In the top panel, the vertical bars on the data points represent statistical uncertainties, whereas in the bottom panels, the total uncertainty in data is indicated by the solid yellow band centred at 1 . In the legend, the $\chi^{2}$ per degree of freedom is given to quantify the goodness of fit of the model to the data.

simulation of MPI. Most of the predictions follow a similar trend describing the differential cross sections and area-normalized distributions with a few exceptions.

The PYThIA8 CP5 tune (with MPI) describes the differential cross section measurements within the uncertainty, but deviate (up to 15-20\%) from the measurements in the lower-region of $\Delta_{\text {rel }} p_{\mathrm{T}}\left(\mathrm{Z}, j_{1}\right)$ and $\Delta_{\text {rel }} p_{\mathrm{T}}$ (Z, dijet), where SPS is expected to be dominant. In the case of area-normalized distributions, the prediction from the MG5_aMC + PYTHIA8 CP5 tune (with MPI) describes the shape of the $\Delta_{\text {rel }} p_{\mathrm{T}}\left(j_{1}, j_{2}\right)$ distribution within the uncertainty, but overestimates in the lower-region of $\Delta_{\text {rel }} p_{\mathrm{T}}\left(\mathrm{Z}, j_{1}\right)$ and $\Delta_{\text {rel }} p_{\mathrm{T}}(\mathrm{Z}$, dijet $)$, but underestimate otherwise. The LO calculations with MG5_aMC + PYTHIA8 provide a similar agreement as obtained by the NLO calculation. The MG5_aMC + HERWIG7 prediction describes the measurements within the uncertainty except some deviations in describing the shapes of the $\Delta \phi\left(\mathrm{Z}, j_{1}\right)$ and $\Delta_{\text {rel }} p_{\mathrm{T}}\left(j_{1}, j_{2}\right)$ distributions. The SHERPA prediction is in reasonable agreement with the measurements within the uncertainty. 

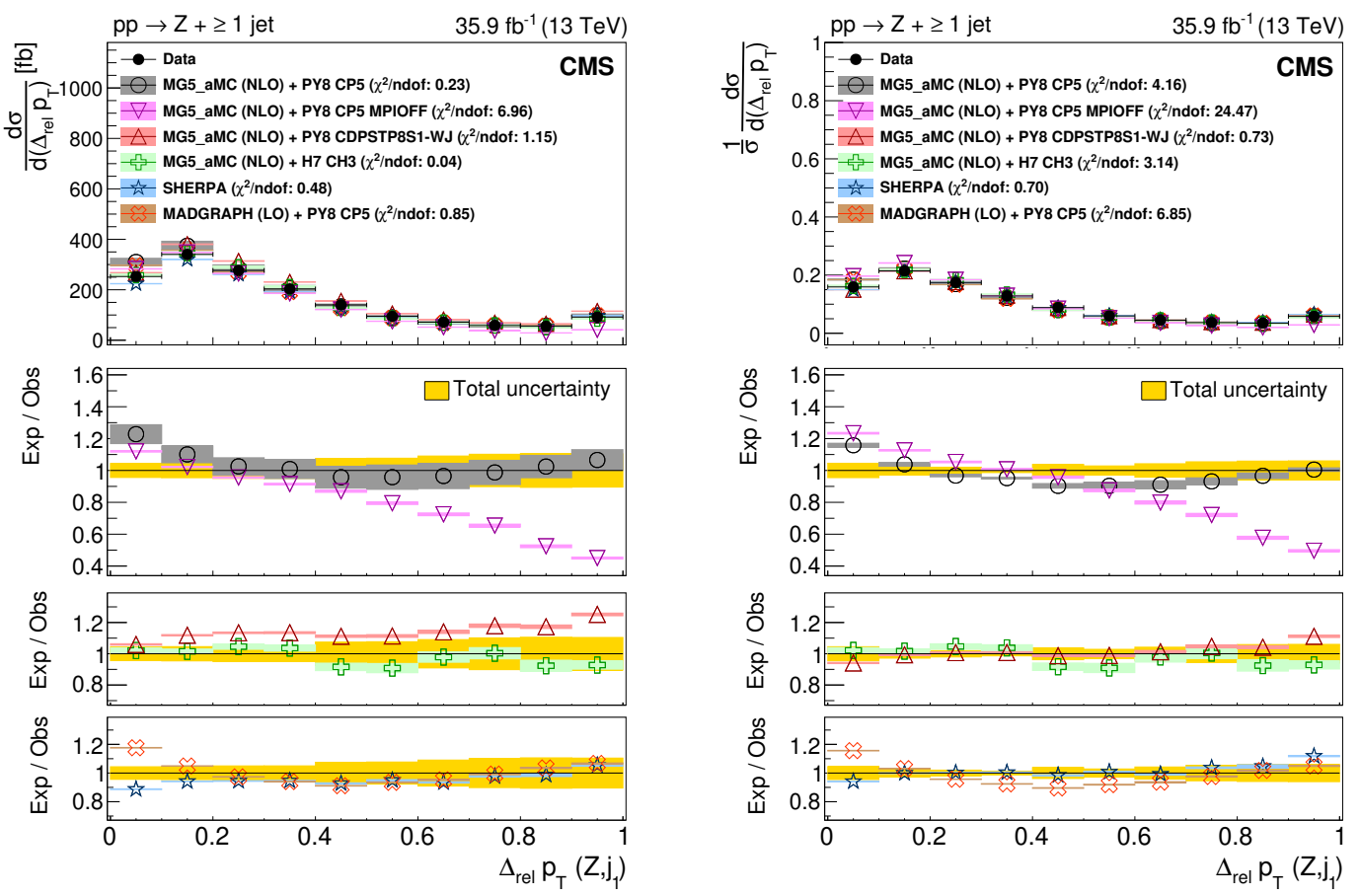

Figure 3. Differential cross sections (left) and area-normalized distributions (right) as functions of the $p_{\mathrm{T}}$ imbalance between the $\mathrm{Z}$ boson and the leading jet for $\mathrm{Z}+\geq 1$ jet events. The uncertainties in the predictions are shown as coloured bands around the theoretical predictions including statistical, PDF, scale, and tune uncertainties for the NLO MG5_aMC + PYTHIA8 (with CP5 tune) and the statistical uncertainty only for the LO MG5_aMC + PYTHIA8 (with CP5 tune), NLO MG5_aMC + PYTHIA8 (with CDPSTP8S1-WJ tune, CP5 tune with MPI-OFF), NLO MG5_aMC + HERWIG7 (with tune CH3), and SHERPA predictions. In the top panel, the vertical bars on the data points represent statistical uncertainties, whereas in the bottom panels, the total uncertainty in data is indicated by the solid yellow band centred at 1 . In the legend, the $\chi^{2}$ per degree of freedom is given to quantify the goodness of fit of the model to the data.

The predictions based on the DPS-specific tune CDPSTP8S1-WJ describe the shape of the distributions within the uncertainty. Since the parameters were derived by fitting only $7 \mathrm{TeV}$ measurements, this suggests that the collision energy dependence of the MPI parameters is well modelled in this tune.

\section{Summary}

The CMS Collaboration has measured the differential cross sections for $\mathrm{Z}+\geq 1$ jet and $\mathrm{Z}+\geq 2$ jet events using proton-proton collision data at $\sqrt{s}=13 \mathrm{TeV}$, corresponding to an integrated luminosity of $35.9 \mathrm{fb}^{-1}$ collected in the year 2016 . The $\mathrm{Z}$ boson is reconstructed using the dimuon channel. This is the first measurement performed to explore observables sensitive to the presence of multi-parton interaction (MPI) using the $\mathrm{Z}+$ jets process at $13 \mathrm{TeV}$. Within the fiducial region, the production cross sections of $\mathrm{Z}+\geq 1$ jet and $\mathrm{Z}+$ $\geq 2$ jet events are measured to be $158.5 \pm 0.3$ (stat) \pm 7.0 (syst) \pm 1.2 (theo) \pm 4.0 (lumi) pb and $44.8 \pm 0.4$ (stat) \pm 3.7 (syst) \pm 0.5 (theo) \pm 1.1 (lumi) pb, respectively. The measured integrated cross section in the fiducial region with jets is well described by the event gen- 

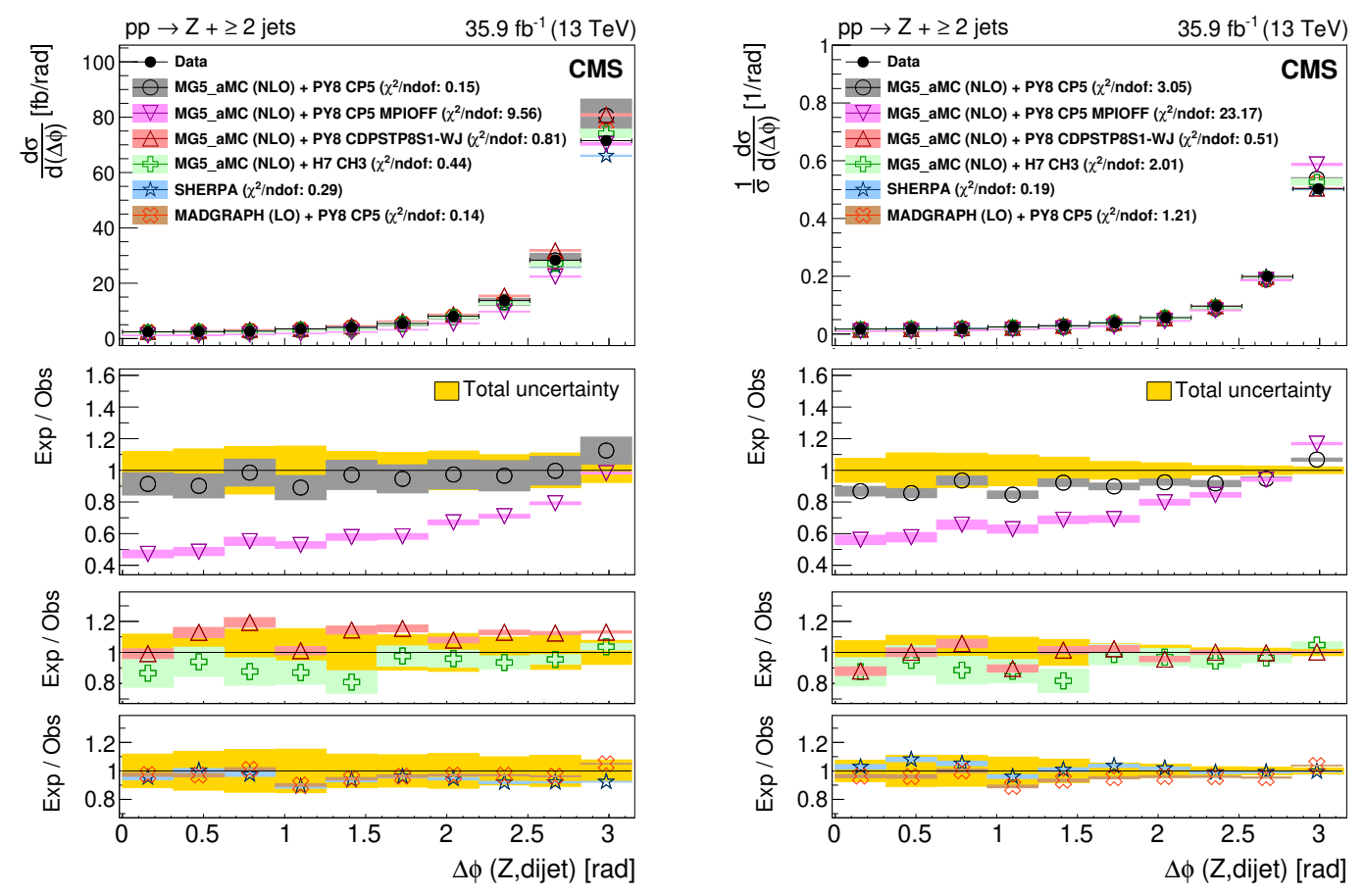

Figure 4. Differential cross sections (left) and area-normalized distributions (right) as functions of $\Delta \phi$ between the $\mathrm{Z}$ boson and the dijet for $\mathrm{Z}+\geq 2$ jet events. The uncertainties in the predictions are shown as coloured bands around the theoretical predictions including statistical, PDF, scale and tune uncertainties for the NLO MG5_aMC + PYTHIA8 (with CP5 tune) and the statistical uncertainty only for the LO MG5_aMC + PYTHIA8 (with CP5 tune), NLO MG5_aMC + PYTHIA8 (with CDPSTP8S1-WJ tune, CP5 tune with MPI-OFF), NLO MG5_aMC + HERWIG7 (with tune $\mathrm{CH} 3$ ), and SHERPA predictions. In the top panel, the vertical bars on the data points represent statistical uncertainties, whereas in the bottom panels, the total uncertainty in data is indicated by the solid yellow band centred at 1 . In the legend, the $\chi^{2}$ per degree of freedom is given to quantify the goodness of fit of the model to the data.

erators SHERPA, MG5_aMC + PYTHIA8, and MG5_aMC + HERWIG7 predictions. The prediction obtained with MG5_aMC + PYTHIA8 with the double-parton scattering (DPS) specific tune CDPSTP8S1-WJ overestimates the measurements by $10-15 \%$, but correctly describes the shape of all the observables. The prediction from MG5_aMC + PYTHIA 8 with the CP5 tune, derived by fitting soft quantum chromodynamics (QCD) measurements, describes the differential cross section and area-normalized distributions. However, there are parts of the distributions that are not well described, such as single parton scattering that dominates lower regions of transverse momentum imbalance $\Delta_{\text {rel }} p_{\mathrm{T}}$ distributions. Predictions with other MPI models describe the measurements well (SHERPA) or reasonably well (MG5_aMC + HERWIG7) except a few deviations in describing the shapes of the $\Delta \phi\left(\mathrm{Z}, j_{1}\right)$ and $\Delta_{\text {rel }} p_{\mathrm{T}}\left(j_{1}, j_{2}\right)$ distributions. The measured distributions show a significant sensitivity to MPI. A proper simulation of MPI is essential to describe the shape of the measured distributions and hence these results are a useful input to further improve DPS-specific tunes and a global tune in combination with other soft QCD measurements. 

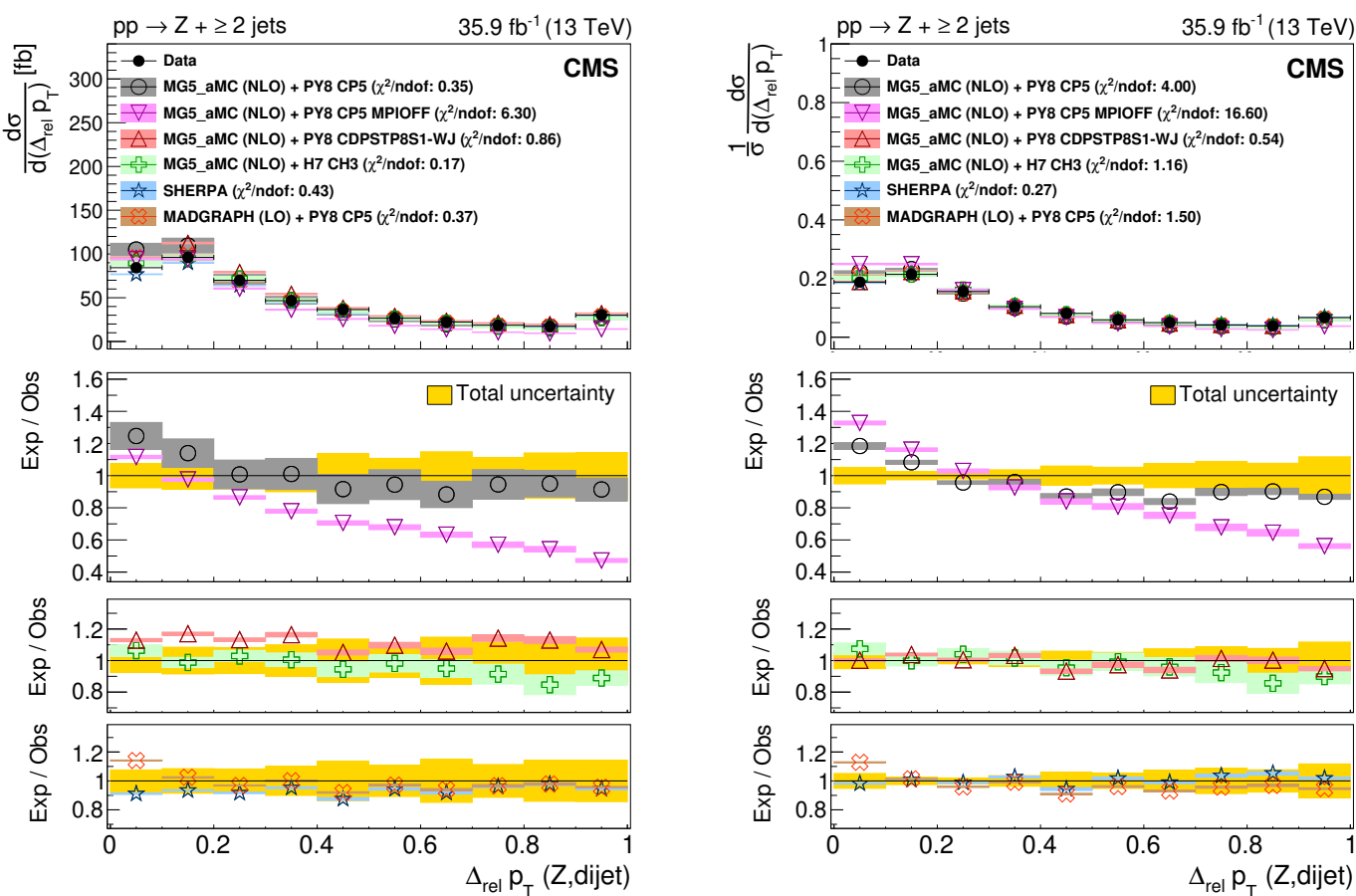

Figure 5. Differential cross sections (left) and area-normalized distributions (right) as functions of the $p_{\mathrm{T}}$ imbalance between the $\mathrm{Z}$ boson and the dijet for $\mathrm{Z}+\geq 2$ jet events. The uncertainties in the predictions are shown as coloured bands around the theoretical predictions including statistical, PDF, scale, and tune uncertainties for the NLO MG5_aMC + PYTHIA8 (with CP5 tune) and the statistical uncertainty only for the LO MG5_aMC + PYTHIA8 (with CP5 tune), NLO MG5_aMC + PYTHIA8 (with CDPSTP8S1-WJ tune, CP5 tune with MPI-OFF), NLO MG5_aMC + HERWIG7 (with tune CH3), and SHERPA predictions. In the top panel, the vertical bars on the data points represent statistical uncertainties, whereas in the bottom panels, the total uncertainty in data is indicated by the solid yellow band centred at 1 . In the legend, the $\chi^{2}$ per degree of freedom is given to quantify the goodness of fit of the model to the data.

\section{Acknowledgments}

We congratulate our colleagues in the CERN accelerator departments for the excellent performance of the LHC and thank the technical and administrative staffs at CERN and at other CMS institutes for their contributions to the success of the CMS effort. In addition, we gratefully acknowledge the computing centres and personnel of the Worldwide LHC Computing Grid and other centres for delivering so effectively the computing infrastructure essential to our analyses. Finally, we acknowledge the enduring support for the construction and operation of the LHC, the CMS detector, and the supporting computing infrastructure provided by the following funding agencies: BMBWF and FWF (Austria); FNRS and FWO (Belgium); CNPq, CAPES, FAPERJ, FAPERGS, and FAPESP (Brazil); MES (Bulgaria); CERN; CAS, MoST, and NSFC (China); MINCIENCIAS (Colombia); MSES and CSF (Croatia); RIF (Cyprus); SENESCYT (Ecuador); MoER, ERC PUT and ERDF (Estonia); Academy of Finland, MEC, and HIP (Finland); CEA and CNRS/IN2P3 (France); BMBF, DFG, and HGF (Germany); GSRT (Greece); NKFIA (Hungary); DAE 

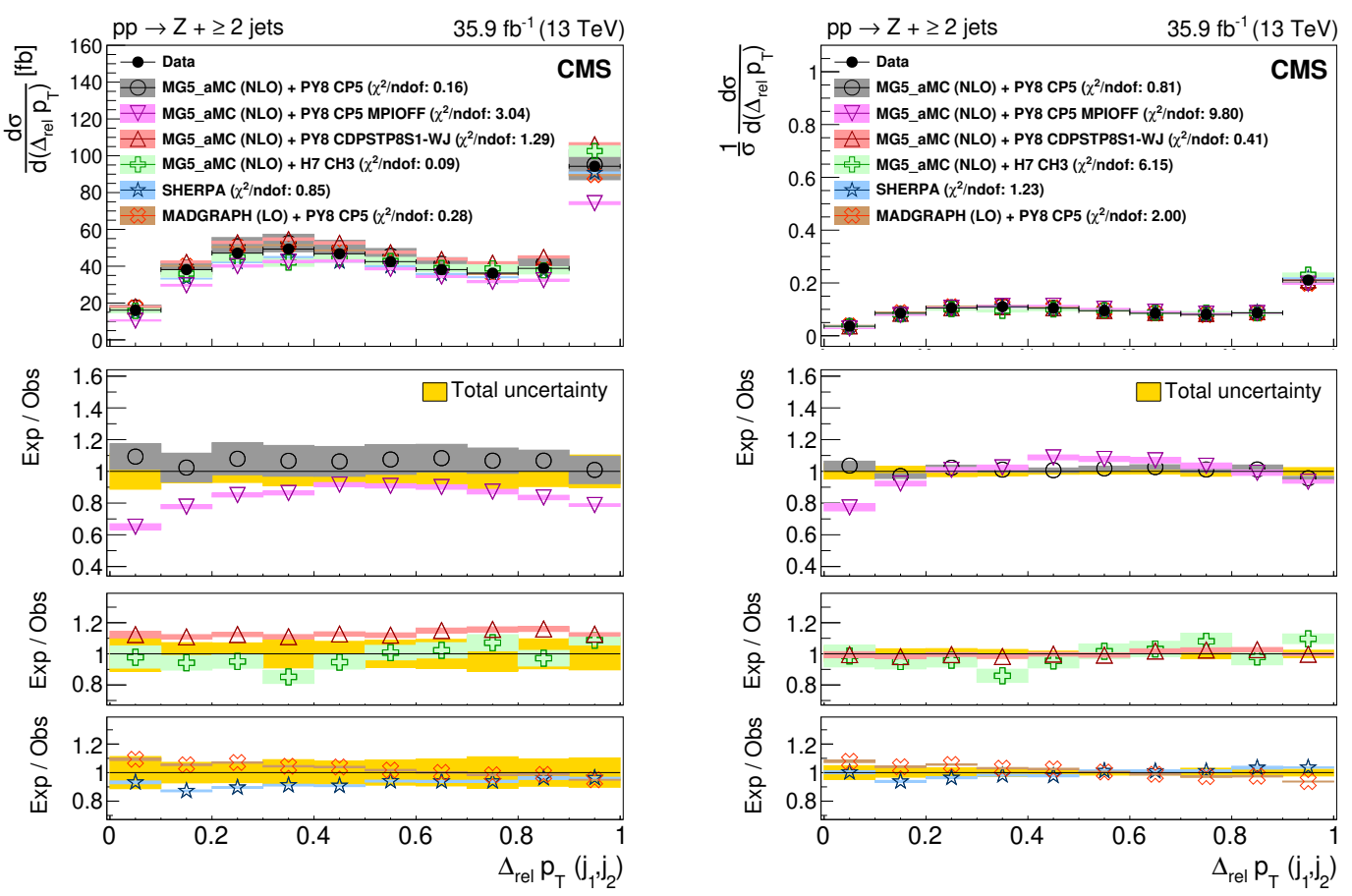

Figure 6. Differential cross sections (left) and area-normalized distributions (right) as functions of the $p_{\mathrm{T}}$ imbalance between leading and subleading jets for $\mathrm{Z}+\geq 2$ jet events. The uncertainties in the predictions are shown as coloured bands around the theoretical predictions including statistical, PDF, scale, and tune uncertainties for the NLO MG5_aMC + PYTHIA8 (with CP5 tune) and the statistical uncertainty only for the LO MG5_aMC + PYTHIA8 (with CP5 tune), NLO MG5_aMC + PYTHIA8 (with CDPSTP8S1-WJ tune, CP5 tune with MPI-OFF), NLO MG5_aMC + HERWIG7 (with tune CH3), and SHERPA predictions. In the top panel, the vertical bars on the data points represent statistical uncertainties, whereas in the bottom panels, the total uncertainty in data is indicated by the solid yellow band centred at 1 . In the legend, the $\chi^{2}$ per degree of freedom is given to quantify the goodness of fit of the model to the data.

and DST (India); IPM (Iran); SFI (Ireland); INFN (Italy); MSIP and NRF (Republic of Korea); MES (Latvia); LAS (Lithuania); MOE and UM (Malaysia); BUAP, CINVESTAV, CONACYT, LNS, SEP, and UASLP-FAI (Mexico); MOS (Montenegro); MBIE (New Zealand); PAEC (Pakistan); MSHE and NSC (Poland); FCT (Portugal); JINR (Dubna); MON, RosAtom, RAS, RFBR, and NRC KI (Russia); MESTD (Serbia); SEIDI, CPAN, PCTI, and FEDER (Spain); MOSTR (Sri Lanka); Swiss Funding Agencies (Switzerland); MST (Taipei); ThEPCenter, IPST, STAR, and NSTDA (Thailand); TUBITAK and TAEK (Turkey); NASU (Ukraine); STFC (United Kingdom); DOE and NSF (U.S.A.).

Individuals have received support from the Marie-Curie programme and the European Research Council and Horizon 2020 Grant, contract Nos. 675440, 724704, 752730, 765710 and 824093 (European Union); the Leventis Foundation; the Alfred P. Sloan Foundation; the Alexander von Humboldt Foundation; the Belgian Federal Science Policy Office; the Fonds pour la Formation à la Recherche dans l'Industrie et dans l'Agriculture (FRIA-Belgium); the Agentschap voor Innovatie door Wetenschap en Technologie (IWT- 
Belgium); the F.R.S.-FNRS and FWO (Belgium) under the "Excellence of Science - EOS" — be.h project n. 30820817; the Beijing Municipal Science \& Technology Commission, No. Z191100007219010; the Ministry of Education, Youth and Sports (MEYS) of the Czech Republic; the Deutsche Forschungsgemeinschaft (DFG), under Germany's Excellence Strategy - EXC 2121 "Quantum Universe" - 390833306, and under project number 400140256 — GRK2497; the Lendület ("Momentum") Programme and the János Bolyai Research Scholarship of the Hungarian Academy of Sciences, the New National Excellence Program ÚNKP, the NKFIA research grants 123842, 123959, 124845, 124850, 125105, 128713, 128786, and 129058 (Hungary); the Council of Science and Industrial Research, India; the Latvian Council of Science; the Ministry of Science and Higher Education and the National Science Center, contracts Opus 2014/15/B/ST2/03998 and 2015/19/B/ST2/02861 (Poland); the National Priorities Research Program by Qatar National Research Fund; the Ministry of Science and Higher Education, project no. 0723-2020-0041 (Russia); the Programa Estatal de Fomento de la Investigación Científica y Técnica de Excelencia María de Maeztu, grant MDM-2015-0509 and the Programa Severo Ochoa del Principado de Asturias; the Thalis and Aristeia programmes cofinanced by EU-ESF and the Greek NSRF; the Rachadapisek Sompot Fund for Postdoctoral Fellowship, Chulalongkorn University and the Chulalongkorn Academic into Its 2nd Century Project Advancement Project (Thailand); the Kavli Foundation; the Nvidia Corporation; the SuperMicro Corporation; the Welch Foundation, contract C-1845; and the Weston Havens Foundation (U.S.A.).

Open Access. This article is distributed under the terms of the Creative Commons Attribution License (CC-BY 4.0), which permits any use, distribution and reproduction in any medium, provided the original author(s) and source are credited.

\section{References}

[1] ATLAS collaboration, Measurement of the production cross section of jets in association with a $Z$ boson in pp collisions at $\sqrt{s}=7$ TeV with the ATLAS detector, JHEP 07 (2013) 032 [arXiv: 1304.7098] [INSPIRE].

[2] ATLAS collaboration, Measurement of the production cross section for $Z / \gamma^{*}$ in association with jets in pp collisions at $\sqrt{s}=7 \mathrm{TeV}$ with the ATLAS detector, Phys. Rev. D 85 (2012) 032009 [arXiv: 1111.2690] [INSPIRE].

[3] CMS collaboration, Jet Production Rates in Association with $W$ and $Z$ Bosons in $p p$ Collisions at $\sqrt{s}=7 \mathrm{TeV}$, JHEP 01 (2012) 010 [arXiv:1110.3226] [INSPIRE].

[4] CMS collaboration, Measurements of jet multiplicity and differential production cross sections of $Z+$ jets events in proton-proton collisions at $\sqrt{s}=7 \mathrm{TeV}$, Phys. Rev. D 91 (2015) 052008 [arXiv: 1408.3104 ] [INSPIRE].

[5] CMS collaboration, Event shapes and azimuthal correlations in $Z+$ jets events in $p p$ collisions at $\sqrt{s}=7 \mathrm{TeV}$, Phys. Lett. B 722 (2013) 238 [arXiv:1301.1646] [InSPIRE].

[6] LHCb collaboration, Study of forward $Z+$ jet production in pp collisions at $\sqrt{s}=7 \mathrm{TeV}$, JHEP 01 (2014) 033 [arXiv:1310.8197] [INSPIRE]. 
[7] CMS collaboration, Comparison of the $Z / \gamma^{*}+$ jets to $\gamma+$ jets cross sections in pp collisions at $\sqrt{s}=8 \mathrm{TeV}$, JHEP 10 (2015) 128 [Erratum ibid. 04 (2016) 010] [arXiv:1505.06520] [INSPIRE].

[8] CMS collaboration, Measurements of differential production cross sections for a $Z$ boson in association with jets in pp collisions at $\sqrt{s}=8 \mathrm{TeV}$, JHEP 04 (2017) 022 [arXiv: 1611.03844] [INSPIRE].

[9] LHCb collaboration, Measurement of forward $W$ and $Z$ boson production in association with jets in proton-proton collisions at $\sqrt{s}=8 \mathrm{TeV}$, JHEP 05 (2016) 131 [arXiv:1605.00951] [INSPIRE].

[10] CMS collaboration, Measurement of differential cross sections for $Z$ boson production in association with jets in proton-proton collisions at $\sqrt{s}=13$ TeV, Eur. Phys. J. C 78 (2018) 965 [arXiv: 1804.05252] [INSPIRE].

[11] ATLAS collaboration, Measurements of the production cross-section for a $Z$ boson in association with b-jets in proton-proton collisions at $\sqrt{s}=13$ TeV with the ATLAS detector, JHEP 07 (2020) 044 [arXiv:2003.11960] [INSPIRE].

[12] CMS collaboration, Constraints on the double-parton scattering cross section from same-sign $W$ boson pair production in proton-proton collisions at $\sqrt{s}=8 \mathrm{TeV}$, JHEP 02 (2018) 032 [arXiv: 1712.02280] [INSPIRE].

[13] ATLAS collaboration, Measurement of hard double-parton interactions in $W(\rightarrow l \nu)+2$ jet events at $\sqrt{s}=7 \mathrm{TeV}$ with the ATLAS detector, New J. Phys. 15 (2013) 033038 [arXiv:1301.6872] [INSPIRE].

[14] CMS collaboration, Study of double parton scattering using $W+2$-jet events in proton-proton collisions at $\sqrt{s}=7 \mathrm{TeV}$, JHEP 03 (2014) 032 [arXiv:1312.5729] [INSPIRE].

[15] ATLAS collaboration, Study of the hard double-parton scattering contribution to inclusive four-lepton production in pp collisions at $\sqrt{s}=8 \mathrm{TeV}$ with the ATLAS detector, Phys. Lett. B 790 (2019) 595 [arXiv:1811.11094] [INSPIRE].

[16] ATLAS collaboration, Observation and measurements of the production of prompt and non-prompt $J / \psi$ mesons in association with a $Z$ boson in pp collisions at $\sqrt{s}=8$ TeV with the ATLAS detector, Eur. Phys. J. C 75 (2015) 229 [arXiv:1412.6428] [InSPIRE].

[17] LHCb collaboration, Observation of $\mathrm{J} / \psi$ pair production in pp collisions at $\sqrt{s}=7 \mathrm{TeV}$, Phys. Lett. B 707 (2012) 52 [arXiv:1109.0963] [InSPIRE].

[18] LHCb collaboration, Measurement of the $J / \psi$ pair production cross-section in pp collisions at $\sqrt{s}=13 \mathrm{TeV}$, JHEP 06 (2017) 047 [Erratum ibid. 10 (2017) 068] [arXiv: 1612.07451] [INSPIRE].

[19] ALICE collaboration, $J / \psi$ production as a function of charged particle multiplicity in $p p$ collisions at $\sqrt{s}=7 \mathrm{TeV}$, Phys. Lett. B 712 (2012) 165 [arXiv:1202.2816] [INSPIRE].

[20] LHCb collaboration, Observation of double charm production involving open charm in pp collisions at $\sqrt{s}=7 \mathrm{TeV}$, JHEP 06 (2012) 141 [Addendum ibid. 03 (2014) 108] [arXiv: 1205.0975] [INSPIRE].

[21] LHCb collaboration, Observation of enhanced double parton scattering in proton-lead collisions at $\sqrt{s_{N N}}=8.16 \mathrm{TeV}$, Phys. Rev. Lett. 125 (2020) 212001 [arXiv:2007.06945] [INSPIRE]. 
[22] D0 collaboration, Double parton interactions in $\gamma+3$ jet events in $p^{-}$bar collisions $\sqrt{s}=1.96 \mathrm{TeV}$, Phys. Rev. D 81 (2010) 052012 [arXiv:0912.5104] [INSPIRE].

[23] CMS collaboration, Extraction and validation of a new set of CMS PYTHIA8 tunes from underlying-event measurements, Eur. Phys. J. C 80 (2020) 4 [arXiv:1903.12179] [INSPIRE].

[24] CMS collaboration, Event generator tunes obtained from underlying event and multiparton scattering measurements, Eur. Phys. J. C 76 (2016) 155 [arXiv:1512.00815] [INSPIRE].

[25] HEPData record for this analysis, http://dx.doi.org/10.17182/hepdata.110665 (2021).

[26] CMS collaboration, Performance of the CMS muon detector and muon reconstruction with proton-proton collisions at $\sqrt{s}=13 \mathrm{TeV}, 2018$ JINST $13 \mathrm{P} 06015$ [arXiv:1804.04528] [INSPIRE].

[27] CMS collaboration, Performance of the CMS Level-1 trigger in proton-proton collisions at $\sqrt{s}=13 \mathrm{TeV}, 2020$ JINST 15 P10017 [arXiv: 2006. 10165] [INSPIRE].

[28] CMS collaboration, The CMS trigger system, 2017 JINST 12 P01020 [arXiv:1609. 02366] [INSPIRE].

[29] CMS collaboration, The CMS experiment at the CERN LHC, 2008 JINST 3 S08004 [INSPIRE].

[30] J. Alwall et al., Comparative study of various algorithms for the merging of parton showers and matrix elements in hadronic collisions, Eur. Phys. J. C 53 (2008) 473 [arXiv:0706.2569] [INSPIRE].

[31] NNPDF collaboration, Parton distributions for the LHC Run II, JHEP 04 (2015) 040 [arXiv: 1410.8849$]$ [INSPIRE].

[32] SherPa collaboration, Event generation with Sherpa 2.2, SciPost Phys. 7 (2019) 034 [arXiv: 1905.09127] [INSPIRE].

[33] S. Hoeche, F. Krauss, M. Schonherr and F. Siegert, QCD matrix elements + parton showers: The NLO case, JHEP 04 (2013) 027 [arXiv: 1207.5030] [INSPIRE].

[34] T. Gehrmann, S. Hoche, F. Krauss, M. Schonherr and F. Siegert, NLO QCD matrix elements + parton showers in $e^{+} e^{-} \rightarrow$ hadrons, JHEP 01 (2013) 144 [arXiv:1207.5031] [InSPIRE].

[35] S. Hoeche, F. Krauss, S. Pozzorini, M. Schoenherr, J.M. Thompson and K.C. Zapp, Triple vector boson production through Higgs-Strahlung with NLO multijet merging, Phys. Rev. D 89 (2014) 093015 [arXiv: 1403.7516] [INSPIRE].

[36] T. Sjöstrand et al., An introduction to PYTHIA 8.2, Comput. Phys. Commun. 191 (2015) 159 [arXiv: 1410.3012] [INSPIRE].

[37] M. Czakon, P. Fiedler and A. Mitov, Total top-quark pair-production cross section at hadron colliders through $O\left(\alpha_{S}^{4}\right)$, Phys. Rev. Lett. 110 (2013) 252004 [arXiv:1303.6254] [INSPIRE].

[38] P. Bärnreuther, M. Czakon and A. Mitov, Percent level precision physics at the Tevatron: first genuine NNLO QCD corrections to $q \bar{q} \rightarrow t \bar{t}+X$, Phys. Rev. Lett. 109 (2012) 132001 [arXiv: 1204.5201] [INSPIRE].

[39] M. Beneke, P. Falgari, S. Klein and C. Schwinn, Hadronic top-quark pair production with NNLL threshold resummation, Nucl. Phys. B 855 (2012) 695 [arXiv:1109.1536] [INSPIRE].

[40] M. Cacciari, M. Czakon, M. Mangano, A. Mitov and P. Nason, Top-pair production at hadron colliders with next-to-next-to-leading logarithmic soft-gluon resummation, Phys. Lett. B 710 (2012) 612 [arXiv:1111.5869] [INSPIRE]. 
[41] M. Czakon and A. Mitov, NNLO corrections to top-pair production at hadron colliders: the all-fermionic scattering channels, JHEP 12 (2012) 054 [arXiv:1207.0236] [INSPIRE].

[42] M. Czakon and A. Mitov, NNLO corrections to top pair production at hadron colliders: the quark-gluon reaction, JHEP 01 (2013) 080 [arXiv: 1210.6832] [INSPIRE].

[43] M. Czakon and A. Mitov, Top++: a program for the calculation of the top-pair cross-section at hadron colliders, Comput. Phys. Commun. 185 (2014) 2930 [arXiv:1112.5675] [INSPIRE].

[44] J.M. Campbell, R.K. Ellis and C. Williams, Vector boson pair production at the LHC, JHEP 07 (2011) 018 [arXiv: 1105.0020] [INSPIRE].

[45] N. Kidonakis, Top quark production, in the proceedings of the Helmholtz International Summer School on Physics of Heavy Quarks and Hadrons, July 15-28, Dubna, Russia (2013) [arXiv: 1311.0283] [INSPIRE].

[46] CMS collaboration, Investigations of the impact of the parton shower tuning in PYTHIA 8 in the modelling of $t \bar{t}$ at $\sqrt{s}=8$ and $13 \mathrm{TeV}$, CMS-PAS-TOP-16-021 (2016).

[47] R.D. Ball et al., Parton distributions with LHC data, Nucl. Phys. B 867 (2013) 244 [arXiv: 1207.1303] [INSPIRE].

[48] J. Alwall, M. Herquet, F. Maltoni, O. Mattelaer and T. Stelzer, MadGraph 5: going beyond, JHEP 06 (2011) 128 [arXiv:1106.0522] [INSPIRE].

[49] J. Alwall, S. de Visscher and F. Maltoni, QCD radiation in the production of heavy colored particles at the LHC, JHEP 02 (2009) 017 [arXiv:0810.5350] [INSPIRE].

[50] R. Frederix and S. Frixione, Merging meets matching in MC@NLO, JHEP 12 (2012) 061 [arXiv:1209.6215] [INSPIRE].

[51] GEANT4 collaboration, GEANT4 - a simulation toolkit, Nucl. Instrum. Meth. A 506 (2003) 250 [INSPIRE].

[52] T. Sjöstrand and M. van Zijl, A multiple interaction model for the event structure in hadron collisions, Phys. Rev. D 36 (1987) 2019 [InSPIRE].

[53] M. Bahr et al., HERWIG++ physics and manual, Eur. Phys. J. C 58 (2008) 639 [arXiv: 0803.0883] [INSPIRE].

[54] J. Bellm et al., HERWIG 7.0/HERWIG++ 3.0 release note, Eur. Phys. J. C 76 (2016) 196 [arXiv: 1512.01178] [INSPIRE].

[55] J. Bellm et al., HERWIG 7.1 release note, arXiv:1705.06919 [INSPIRE].

[56] CMS collaboration, Development and validation of HERWIG 7 tunes from CMS underlying-event measurements, Eur. Phys. J. C 81 (2021) 312 [arXiv:2011.03422] [INSPIRE].

[57] K. Melnikov and F. Petriello, Electroweak gauge boson production at hadron colliders through $O\left(\alpha_{s}^{2}\right)$, Phys. Rev. D 74 (2006) 114017 [hep-ph/0609070] [INSPIRE].

[58] CMS collaboration, Particle-flow reconstruction and global event description with the CMS detector, 2017 JINST 12 P10003 [arXiv:1706.04965] [INSPIRE].

[59] M. Cacciari, G.P. Salam and G. Soyez, The anti- $k_{t}$ jet clustering algorithm, JHEP 04 (2008) 063 [arXiv: 0802.1189] [INSPIRE].

[60] M. Cacciari, G.P. Salam and G. Soyez, FastJet user manual, Eur. Phys. J. C 72 (2012) 1896 [arXiv: 1111.6097] [INSPIRE]. 
[61] CMS collaboration, Measurement of the inclusive $W$ and $Z$ production cross sections in $p p$ collisions at $\sqrt{s}=7 \mathrm{TeV}$, JHEP 10 (2011) 132 [arXiv:1107.4789] [INSPIRE].

[62] M. Cacciari and G.P. Salam, Pileup subtraction using jet areas, Phys. Lett. B 659 (2008) 119 [arXiv: 0707.1378] [INSPIRE].

[63] CMS collaboration, Jet energy scale and resolution in the CMS experiment in pp collisions at $8 \mathrm{TeV}, 2017$ JINST $12 \mathrm{P} 02014$ [arXiv: 1607. 03663] [INSPIRE].

[64] CMS collaboration, Jet energy scale and resolution performance with $13 \mathrm{TeV}$ data collected by CMS in 2016, CMS-DP-2018-028 (2018).

[65] CMS collaboration, Jet performance in pp collisions at 7 TeV, CMS-PAS-JME-10-003 (2010).

[66] CMS collaboration, Pileup jet identification, CMS-PAS-JME-13-005 (2013).

[67] CMS collaboration, Pileup mitigation at CMS in 13 TeV data, 2020 JINST 15 P09018 [arXiv: 2003. 00503] [INSPIRE].

[68] CMS collaboration, Electron and photon reconstruction and identification with the CMS experiment at the CERN LHC, 2021 JINST 16 P05014 [arXiv:2012.06888] [INSPIRE].

[69] S. Schmitt, TUnfold: an algorithm for correcting migration effects in high energy physics, 2012 JINST 7 T10003 [arXiv:1205.6201] [INSPIRE].

[70] A.N. Tikhonov, Solution of incorrectly formulated problems and the regularization method, Soviet Math. Dokl. 4 (1963) 1035.

[71] CMS collaboration, Object definitions for top quark analyses at the particle level, CMS-NOTE-2006-106 (2017).

[72] J. Butterworth et al., PDF 4LHC recommendations for LHC Run II, J. Phys. G 43 (2016) 023001 [arXiv: 1510.03865] [INSPIRE].

[73] CMS collaboration, Precision luminosity measurement in proton-proton collisions at $\sqrt{s}=13 \mathrm{TeV}$ in 2015 and 2016 at CMS, Eur. Phys. J. C 81 (2021) 800 [arXiv:2104.01927] [INSPIRE].

[74] CMS collaboration, Measurement of the inelastic proton-proton cross section at $\sqrt{s}=13 \mathrm{TeV}$, JHEP 07 (2018) 161 [arXiv: 1802.02613] [INSPIRE]. 


\section{The CMS collaboration}

\section{Yerevan Physics Institute, Yerevan, Armenia}

A. Tumasyan

\section{Institut für Hochenergiephysik, Wien, Austria}

W. Adam, J.W. Andrejkovic, T. Bergauer, S. Chatterjee, M. Dragicevic, A. Es-

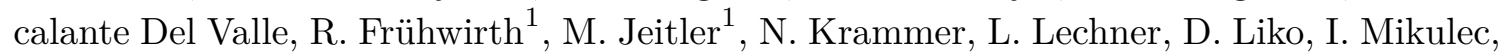
P. Paulitsch, F.M. Pitters, J. Schieck ${ }^{1}$, R. Schöfbeck, M. Spanring, S. Templ, W. Waltenberger, C.-E. Wulz ${ }^{1}$

Institute for Nuclear Problems, Minsk, Belarus

V. Chekhovsky, A. Litomin, V. Makarenko

\section{Universiteit Antwerpen, Antwerpen, Belgium}

M.R. Darwish ${ }^{2}$, E.A. De Wolf, X. Janssen, T. Kello ${ }^{3}$, A. Lelek, H. Rejeb Sfar, P. Van Mechelen, S. Van Putte, N. Van Remortel

\section{Vrije Universiteit Brussel, Brussel, Belgium}

F. Blekman, E.S. Bols, J. D'Hondt, J. De Clercq, M. Delcourt, H. El Faham, S. Lowette, S. Moortgat, A. Morton, D. Müller, A.R. Sahasransu, S. Tavernier, W. Van Doninck, P. Van Mulders

\section{Université Libre de Bruxelles, Bruxelles, Belgium}

D. Beghin, B. Bilin, B. Clerbaux, G. De Lentdecker, L. Favart, A. Grebenyuk, A.K. Kalsi, K. Lee, M. Mahdavikhorrami, I. Makarenko, L. Moureaux, L. Pétré, A. Popov, N. Postiau, E. Starling, L. Thomas, M. Vanden Bemden, C. Vander Velde, P. Vanlaer, D. Vannerom, L. Wezenbeek

\section{Ghent University, Ghent, Belgium}

T. Cornelis, D. Dobur, J. Knolle, L. Lambrecht, G. Mestdach, M. Niedziela, C. Roskas, A. Samalan, K. Skovpen, T.T. Tran, M. Tytgat, W. Verbeke, B. Vermassen, M. Vit

\section{Université Catholique de Louvain, Louvain-la-Neuve, Belgium}

A. Bethani, G. Bruno, F. Bury, C. Caputo, P. David, C. Delaere, I.S. Donertas, A. Giammanco, K. Jaffel, V. Lemaitre, K. Mondal, J. Prisciandaro, A. Taliercio, M. Teklishyn, P. Vischia, S. Wertz, S. Wuyckens

Centro Brasileiro de Pesquisas Fisicas, Rio de Janeiro, Brazil

G.A. Alves, C. Hensel, A. Moraes

Universidade do Estado do Rio de Janeiro, Rio de Janeiro, Brazil

W.L. Aldá Júnior, M. Alves Gallo Pereira, M. Barroso Ferreira Filho, H. BRANDAO MALBOUISSON, W. Carvalho, J. Chinellato ${ }^{4}$, E.M. Da Costa, G.G. Da Silveira ${ }^{5}$, D. De Jesus Damiao, S. Fonseca De Souza, D. Matos Figueiredo, C. Mora Herrera, K. Mota Amarilo, L. Mundim, H. Nogima, P. Rebello Teles, A. Santoro, S.M. Silva Do Amaral, A. Sznajder, M. Thiel, F. Torres Da Silva De Araujo, A. Vilela Pereira 
Universidade Estadual Paulista ${ }^{a}$, Universidade Federal do $\mathrm{ABC}^{b}$, São Paulo, Brazil

C.A. Bernardes ${ }^{a}$, L. Calligaris ${ }^{a}$, T.R. Fernandez Perez Tomei ${ }^{a}$, E.M. Gregores ${ }^{a, b}$, D.S. Lemos ${ }^{a}$, P.G. Mercadante ${ }^{a, b}$, S.F. Novaes ${ }^{a}$, Sandra S. Padula ${ }^{a}$

Institute for Nuclear Research and Nuclear Energy, Bulgarian Academy of Sciences, Sofia, Bulgaria

A. Aleksandrov, G. Antchev, R. Hadjiiska, P. Iaydjiev, M. Misheva, M. Rodozov,

M. Shopova, G. Sultanov

University of Sofia, Sofia, Bulgaria

A. Dimitrov, T. Ivanov, L. Litov, B. Pavlov, P. Petkov, A. Petrov

Beihang University, Beijing, China

T. Cheng, W. Fang ${ }^{3}$, Q. Guo, T. Javaid ${ }^{6}$, M. Mittal, H. Wang, L. Yuan

Department of Physics, Tsinghua University, Beijing, China

M. Ahmad, G. Bauer, C. Dozen ${ }^{7}$, Z. Hu, J. Martins ${ }^{8}$, Y. Wang, K. Yi ${ }^{9,10}$

Institute of High Energy Physics, Beijing, China

E. Chapon, G.M. Chen ${ }^{6}$, H.S. Chen ${ }^{6}$, M. Chen, F. Iemmi, A. Kapoor, D. Leggat, H. Liao, Z.-A. Liu ${ }^{6}$, V. Milosevic, F. Monti, R. Sharma, J. Tao, J. Thomas-Wilsker, J. Wang, H. Zhang, S. Zhang ${ }^{6}$, J. Zhao

State Key Laboratory of Nuclear Physics and Technology, Peking University, Beijing, China

A. Agapitos, Y. Ban, C. Chen, Q. Huang, A. Levin, Q. Li, X. Lyu, Y. Mao, S.J. Qian, D. Wang, Q. Wang, J. Xiao

\section{Sun Yat-Sen University, Guangzhou, China}

M. Lu, Z. You

Institute of Modern Physics and Key Laboratory of Nuclear Physics and Ionbeam Application (MOE) — Fudan University, Shanghai, China

X. $\mathrm{Gao}^{3}, \mathrm{H}$. Okawa

Zhejiang University, Hangzhou, China

Z. Lin, M. Xiao

Universidad de Los Andes, Bogota, Colombia

C. Avila, A. Cabrera, C. Florez, J. Fraga, A. Sarkar, M.A. Segura Delgado

Universidad de Antioquia, Medellin, Colombia

J. Mejia Guisao, F. Ramirez, J.D. Ruiz Alvarez, C.A. Salazar González

University of Split, Faculty of Electrical Engineering, Mechanical Engineering and Naval Architecture, Split, Croatia

D. Giljanovic, N. Godinovic, D. Lelas, I. Puljak 
University of Split, Faculty of Science, Split, Croatia

Z. Antunovic, M. Kovac, T. Sculac

Institute Rudjer Boskovic, Zagreb, Croatia

V. Brigljevic, D. Ferencek, D. Majumder, M. Roguljic, A. Starodumov ${ }^{11}$, T. Susa

University of Cyprus, Nicosia, Cyprus

A. Attikis, E. Erodotou, A. Ioannou, G. Kole, M. Kolosova, S. Konstantinou, J. Mousa,

C. Nicolaou, F. Ptochos, P.A. Razis, H. Rykaczewski, H. Saka

Charles University, Prague, Czech Republic

M. Finger ${ }^{12}$, M. Finger Jr. ${ }^{12}$, A. Kveton

Escuela Politecnica Nacional, Quito, Ecuador

E. Ayala

Universidad San Francisco de Quito, Quito, Ecuador

E. Carrera Jarrin

Academy of Scientific Research and Technology of the Arab Republic of Egypt, Egyptian Network of High Energy Physics, Cairo, Egypt

H. Abdalla ${ }^{13}$, S. Abu Zeid ${ }^{14}$

Center for High Energy Physics (CHEP-FU), Fayoum University, El-Fayoum, Egypt

A. Lotfy, M.A. Mahmoud

National Institute of Chemical Physics and Biophysics, Tallinn, Estonia

S. Bhowmik, A. Carvalho Antunes De Oliveira, R.K. Dewanjee, K. Ehataht, M. Kadastik,

C. Nielsen, J. Pata, M. Raidal, L. Tani, C. Veelken

Department of Physics, University of Helsinki, Helsinki, Finland

P. Eerola, L. Forthomme, H. Kirschenmann, K. Osterberg, M. Voutilainen

Helsinki Institute of Physics, Helsinki, Finland

S. Bharthuar, E. Brücken, F. Garcia, J. Havukainen, M.S. Kim, R. Kinnunen, T. Lampén,

K. Lassila-Perini, S. Lehti, T. Lindén, M. Lotti, L. Martikainen, J. Ott, H. Siikonen,

E. Tuominen, J. Tuominiemi

Lappeenranta University of Technology, Lappeenranta, Finland

P. Luukka, H. Petrow, T. Tuuva

IRFU, CEA, Université Paris-Saclay, Gif-sur-Yvette, France

C. Amendola, M. Besancon, F. Couderc, M. Dejardin, D. Denegri, J.L. Faure, F. Ferri,

S. Ganjour, A. Givernaud, P. Gras, G. Hamel de Monchenault, P. Jarry, B. Lenzi, E. Locci,

J. Malcles, J. Rander, A. Rosowsky, M.Ö. Sahin, A. Savoy-Navarro ${ }^{15}$, M. Titov, G.B. Yu

Laboratoire Leprince-Ringuet, CNRS/IN2P3, Ecole Polytechnique, Institut Polytechnique de Paris, Palaiseau, France

S. Ahuja, F. Beaudette, M. Bonanomi, A. Buchot Perraguin, P. Busson, A. Cappati, C. Charlot, O. Davignon, B. Diab, G. Falmagne, S. Ghosh, R. Granier de Cassagnac, 
A. Hakimi, I. Kucher, M. Nguyen, C. Ochando, P. Paganini, J. Rembser, R. Salerno, J.B. Sauvan, Y. Sirois, A. Zabi, A. Zghiche

\section{Université de Strasbourg, CNRS, IPHC UMR 7178, Strasbourg, France}

J.-L. Agram ${ }^{16}$, J. Andrea, D. Apparu, D. Bloch, G. Bourgatte, J.-M. Brom, E.C. Chabert, C. Collard, D. Darej, J.-C. Fontaine ${ }^{16}$, U. Goerlach, C. Grimault, A.-C. Le Bihan, E. Nibigira, P. Van Hove

Institut de Physique des 2 Infinis de Lyon (IP2I ), Villeurbanne, France

E. Asilar, S. Beauceron, C. Bernet, G. Boudoul, C. Camen, A. Carle, N. Chanon,

D. Contardo, P. Depasse, H. El Mamouni, J. Fay, S. Gascon, M. Gouzevitch, B. Ille,

Sa. Jain, I.B. Laktineh, H. Lattaud, A. Lesauvage, M. Lethuillier, L. Mirabito, S. Perries,

K. Shchablo, V. Sordini, L. Torterotot, G. Touquet, M. Vander Donckt, S. Viret

Georgian Technical University, Tbilisi, Georgia

I. Lomidze, T. Toriashvili ${ }^{17}, \mathrm{Z}$. Tsamalaidze ${ }^{12}$

RWTH Aachen University, I. Physikalisches Institut, Aachen, Germany

L. Feld, K. Klein, M. Lipinski, D. Meuser, A. Pauls, M.P. Rauch, N. Röwert, J. Schulz, M. Teroerde

\section{RWTH Aachen University, III. Physikalisches Institut A, Aachen, Germany}

D. Eliseev, M. Erdmann, P. Fackeldey, B. Fischer, S. Ghosh, T. Hebbeker, K. Hoepfner, F. Ivone, H. Keller, L. Mastrolorenzo, M. Merschmeyer, A. Meyer, G. Mocellin, S. Mondal, S. Mukherjee, D. Noll, A. Novak, T. Pook, A. Pozdnyakov, Y. Rath, H. Reithler, J. Roemer, A. Schmidt, S.C. Schuler, A. Sharma, S. Wiedenbeck, S. Zaleski

RWTH Aachen University, III. Physikalisches Institut B, Aachen, Germany

C. Dziwok, G. Flügge, W. Haj Ahmad ${ }^{18}$, O. Hlushchenko, T. Kress, A. Nowack, C. Pistone, O. Pooth, D. Roy, H. Sert, A. Stahl ${ }^{19}$, T. Ziemons

\section{Deutsches Elektronen-Synchrotron, Hamburg, Germany}

H. Aarup Petersen, M. Aldaya Martin, P. Asmuss, I. Babounikau, S. Baxter, O. Behnke, A. Bermúdez Martínez, A.A. Bin Anuar, K. Borras ${ }^{20}$, V. Botta, D. Brunner, A. Campbell, A. Cardini, C. Cheng, S. Consuegra Rodríguez, G. Correia Silva, V. Danilov, L. Didukh, G. Eckerlin, D. Eckstein, L.I. Estevez Banos, O. Filatov, E. Gallo ${ }^{21}$, A. Geiser, A. Giraldi, A. Grohsjean, M. Guthoff, A. Jafari ${ }^{22}$, N.Z. Jomhari, H. Jung, A. Kasem ${ }^{20}$, M. Kasemann, H. Kaveh, C. Kleinwort, D. Krücker, W. Lange, J. Lidrych, K. Lipka, W. Lohmann ${ }^{23}$, R. Mankel, I.-A. Melzer-Pellmann, J. Metwally, A.B. Meyer, M. Meyer, J. Mnich, A. Mussgiller, Y. Otarid, D. Pérez Adán, D. Pitzl, A. Raspereza, B. Ribeiro Lopes, J. Rübenach, A. Saggio, A. Saibel, M. Savitskyi, M. Scham, V. Scheurer, C. Schwanenberger ${ }^{21}$, A. Singh, R.E. Sosa Ricardo, D. Stafford, N. Tonon, O. Turkot, M. Van De Klundert, R. Walsh, D. Walter, Y. Wen, K. Wichmann, L. Wiens, C. Wissing, S. Wuchterl

\section{University of Hamburg, Hamburg, Germany}

R. Aggleton, S. Bein, L. Benato, A. Benecke, P. Connor, K. De Leo, M. Eich, F. Feindt, A. Fröhlich, C. Garbers, E. Garutti, P. Gunnellini, J. Haller, A. Hinzmann, G. Kasieczka, 
R. Klanner, R. Kogler, T. Kramer, V. Kutzner, J. Lange, T. Lange, A. Lobanov, A. Malara, A. Nigamova, K.J. Pena Rodriguez, O. Rieger, P. Schleper, M. Schröder, J. Schwandt, D. Schwarz, J. Sonneveld, H. Stadie, G. Steinbrück, A. Tews, B. Vormwald, I. Zoi

Karlsruher Institut fuer Technologie, Karlsruhe, Germany

J. Bechtel, T. Berger, E. Butz, R. Caspart, T. Chwalek, W. De Boer ${ }^{\dagger}$, A. Dierlamm, A. Droll, K. El Morabit, N. Faltermann, M. Giffels, J.o. Gosewisch, A. Gottmann, F. Hartmann ${ }^{19}$, C. Heidecker, U. Husemann, I. Katkov ${ }^{24}$, P. Keicher, R. Koppenhöfer, S. Maier, M. Metzler, S. Mitra, Th. Müller, M. Neukum, A. Nürnberg, G. Quast, K. Rabbertz, J. Rauser, D. Savoiu, M. Schnepf, D. Seith, I. Shvetsov, H.J. Simonis, R. Ulrich, J. Van Der Linden, R.F. Von Cube, M. Wassmer, M. Weber, S. Wieland, R. Wolf, S. Wozniewski, S. Wunsch

Institute of Nuclear and Particle Physics (INPP), NCSR Demokritos, Aghia Paraskevi, Greece

G. Anagnostou, P. Asenov, G. Daskalakis, T. Geralis, A. Kyriakis, D. Loukas, A. Stakia

National and Kapodistrian University of Athens, Athens, Greece

M. Diamantopoulou, D. Karasavvas, G. Karathanasis, P. Kontaxakis, C.K. Koraka, A. Manousakis-Katsikakis, A. Panagiotou, I. Papavergou, N. Saoulidou, K. Theofilatos, E. Tziaferi, K. Vellidis, E. Vourliotis

National Technical University of Athens, Athens, Greece

G. Bakas, K. Kousouris, I. Papakrivopoulos, G. Tsipolitis, A. Zacharopoulou

University of Ioánnina, Ioánnina, Greece

I. Evangelou, C. Foudas, P. Gianneios, P. Katsoulis, P. Kokkas, N. Manthos, I. Papadopoulos, J. Strologas

MTA-ELTE Lendület CMS Particle and Nuclear Physics Group, Eötvös Loránd University, Budapest, Hungary

M. Csanad, K. Farkas, M.M.A. Gadallah ${ }^{25}$, S. Lökös ${ }^{26}$, P. Major, K. Mandal, A. Mehta, G. Pasztor, A.J. Rádl, O. Surányi, G.I. Veres

Wigner Research Centre for Physics, Budapest, Hungary

M. Bartók ${ }^{27}$, G. Bencze, C. Hajdu, D. Horvath ${ }^{28}$, F. Sikler, V. Veszpremi, G. Vesztergombi ${ }^{\dagger}$

Institute of Nuclear Research ATOMKI, Debrecen, Hungary

S. Czellar, J. Karancsi ${ }^{27}$, J. Molnar, Z. Szillasi, D. Teyssier

Institute of Physics, University of Debrecen, Debrecen, Hungary

P. Raics, Z.L. Trocsanyi ${ }^{29}$, B. Ujvari

Karoly Robert Campus, MATE Institute of Technology

T. Csorgo ${ }^{30}$, F. Nemes ${ }^{30}$, T. Novak

Indian Institute of Science (IISc), Bangalore, India

J.R. Komaragiri, D. Kumar, L. Panwar, P.C. Tiwari 
National Institute of Science Education and Research, HBNI, Bhubaneswar, India

S. Bahinipati ${ }^{31}$, D. Dash, C. Kar, P. Mal, T. Mishra, V.K. Muraleedharan Nair Bindhu ${ }^{32}$, A. Nayak ${ }^{32}$, P. Saha, N. Sur, S.K. Swain, D. Vats ${ }^{32}$

Panjab University, Chandigarh, India

S. Bansal, S.B. Beri, V. Bhatnagar, G. Chaudhary, S. Chauhan, N. Dhingra ${ }^{33}$, R. Gupta, A. Kaur, M. Kaur, S. Kaur, P. Kumari, M. Meena, K. Sandeep, J.B. Singh, A.K. Virdi

University of Delhi, Delhi, India

A. Ahmed, A. Bhardwaj, B.C. Choudhary, R.B. Garg, M. Gola, S. Keshri, A. Kumar, M. Naimuddin, P. Priyanka, K. Ranjan, A. Shah

Saha Institute of Nuclear Physics, HBNI, Kolkata, India

M. Bharti ${ }^{34}$, R. Bhattacharya, S. Bhattacharya, D. Bhowmik, S. Dutta, S. Dutta, B. Gomber ${ }^{35}$, M. Maity ${ }^{36}$, S. Nandan, P. Palit, P.K. Rout, G. Saha, B. Sahu, S. Sarkar, M. Sharan, B. Singh ${ }^{34}$, S. Thakur ${ }^{34}$

Indian Institute of Technology Madras, Madras, India

P.K. Behera, S.C. Behera, P. Kalbhor, A. Muhammad, R. Pradhan, P.R. Pujahari, A. Sharma, A.K. Sikdar

Bhabha Atomic Research Centre, Mumbai, India

D. Dutta, V. Jha, V. Kumar, D.K. Mishra, K. Naskar ${ }^{37}$, P.K. Netrakanti, L.M. Pant, P. Shukla

Tata Institute of Fundamental Research-A, Mumbai, India

T. Aziz, S. Dugad, M. Kumar, U. Sarkar

Tata Institute of Fundamental Research-B, Mumbai, India

S. Banerjee, S. Bhattacharya, R. Chudasama, M. Guchait, S. Karmakar, S. Kumar, G. Majumder, K. Mazumdar, S. Mukherjee

Indian Institute of Science Education and Research (IISER), Pune, India

K. Alpana, S. Dube, B. Kansal, S. Pandey, A. Rane, A. Rastogi, S. Sharma

Department of Physics, Isfahan University of Technology, Isfahan, Iran

H. Bakhshiansohi ${ }^{38}$, M. Zeinali ${ }^{39}$

Institute for Research in Fundamental Sciences (IPM), Tehran, Iran

S. Chenarani ${ }^{40}$, S.M. Etesami, M. Khakzad, M. Mohammadi Najafabadi

University College Dublin, Dublin, Ireland

M. Grunewald

INFN Sezione di Bari ${ }^{a}$, Università di Bari $^{b}$, Politecnico di Bari ${ }^{c}$, Bari, Italy M. Abbrescia ${ }^{a, b}$, R. Aly ${ }^{a, b, 41}$, C. Aruta ${ }^{a, b}$, A. Colaleo $^{a}$, D. Creanza ${ }^{a, c}$, N. De Filippis ${ }^{a, c}$,

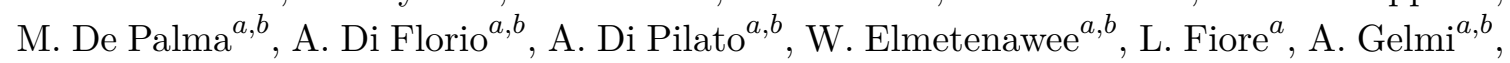

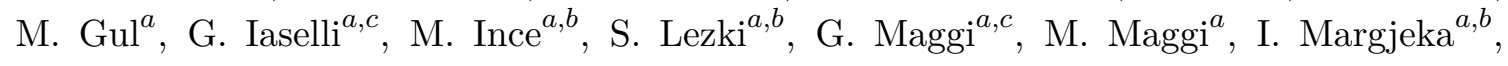




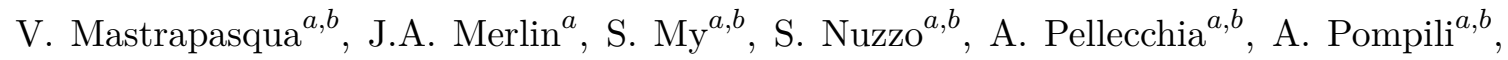

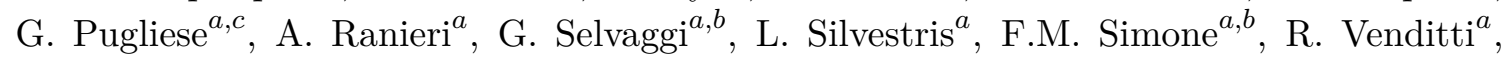
P. Verwilligen ${ }^{a}$

INFN Sezione di Bologna ${ }^{a}$, Università di Bologna ${ }^{b}$, Bologna, Italy

G. Abbiendi $^{a}$, C. Battilana ${ }^{a, b}$, D. Bonacorsi ${ }^{a, b}$, L. Borgonovi ${ }^{a}$, L. Brigliadori ${ }^{a}$, R. Campanini ${ }^{a, b}$, P. Capiluppi ${ }^{a, b}$, A. Castro ${ }^{a, b}$, F.R. Cavallo ${ }^{a}$, M. Cuffiani ${ }^{a, b}$, G.M. Dallavalle ${ }^{a}$, T. Diotalevi ${ }^{a, b}$, F. Fabbri $^{a}$, A. Fanfani ${ }^{a, b}$, P. Giacomelli $^{a}$, L. Giommi $^{a, b}{ }^{,}$ C. Grandi ${ }^{a}$, L. Guiducci ${ }^{a, b}$, S. Lo Meo ${ }^{a, 42}$, L. Lunerti ${ }^{a, b}$, S. Marcellini ${ }^{a}$, G. Masetti ${ }^{a}$,

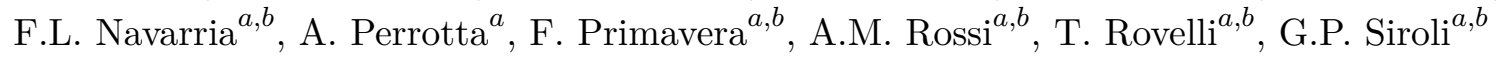

INFN Sezione di Catania ${ }^{a}$, Università di Catania ${ }^{b}$, Catania, Italy

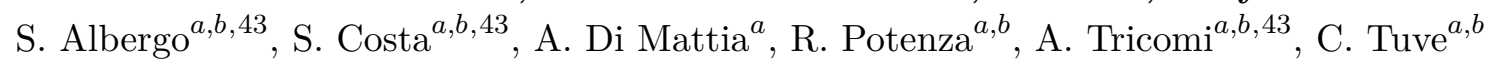

INFN Sezione di Firenze ${ }^{a}$, Università di Firenze ${ }^{b}$, Firenze, Italy

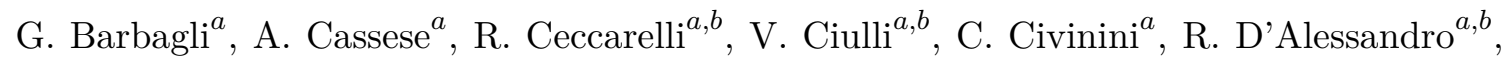

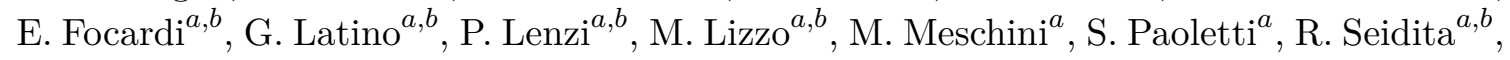

G. Sguazzoni ${ }^{a}$, L. Viliani $^{a}$

INFN Laboratori Nazionali di Frascati, Frascati, Italy

L. Benussi, S. Bianco, D. Piccolo

INFN Sezione di Genova ${ }^{a}$, Università di Genova ${ }^{b}$, Genova, Italy

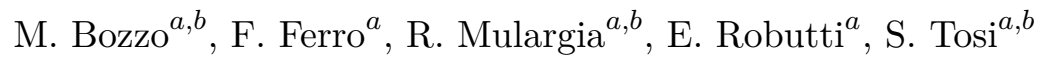

INFN Sezione di Milano-Bicocca $^{a}$, Università di Milano-Bicocca ${ }^{b}$, Milano, Italy

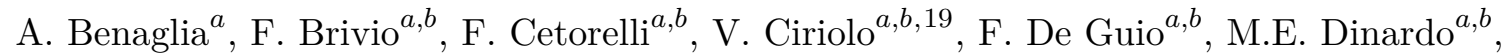
P. Dini ${ }^{a}$, S. Gennai ${ }^{a}$, A. Ghezzi ${ }^{a, b}$, P. Govoni $^{a, b}$, L. Guzzi $^{a}, b$, M. Malberti $^{a}$, S. Malvezzi $^{a}$, A. Massironi ${ }^{a}$, D. Menasce ${ }^{a}$, L. Moroni ${ }^{a}$, M. Paganoni ${ }^{a, b}$, D. Pedrini ${ }^{a}$, S. Ragazzi $^{a, b}$, N. Redaelli ${ }^{a}$, T. Tabarelli de Fatis ${ }^{a, b}$, D. Valsecchi ${ }^{a, b, 19}$, D. Zuolo ${ }^{a, b}$

INFN Sezione di Napoli ${ }^{a}$, Università di Napoli 'Federico II' ${ }^{b}$, Napoli, Italy, Università della Basilicata $^{c}$, Potenza, Italy, Università G. Marconi ${ }^{d}$, Roma, Italy

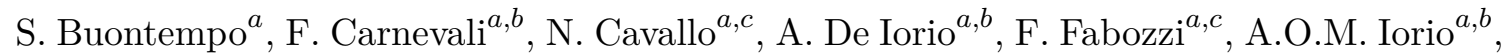

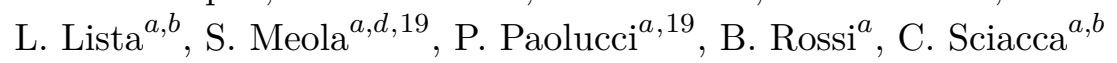

INFN Sezione di Padova ${ }^{a}$, Università di Padova ${ }^{b}$, Padova, Italy, Università di Trento ${ }^{c}$, Trento, Italy

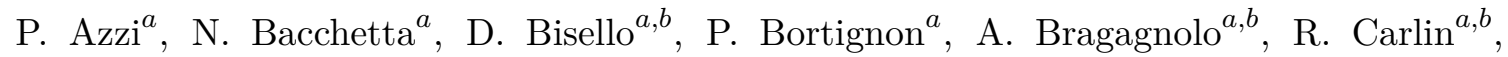

P. Checchia ${ }^{a}$, P. De Castro Manzano ${ }^{a}$, T. Dorigo ${ }^{a}$, U. Dosselli ${ }^{a}$, F. Gasparini ${ }^{a, b}$,

U. Gasparini ${ }^{a, b}$, S.Y. Hoh ${ }^{a, b}$, L. Layer ${ }^{a, 44}$, M. Margoni ${ }^{a, b}$, A.T. Meneguzzo ${ }^{a, b}$, J. Pazzini $^{a, b}$,

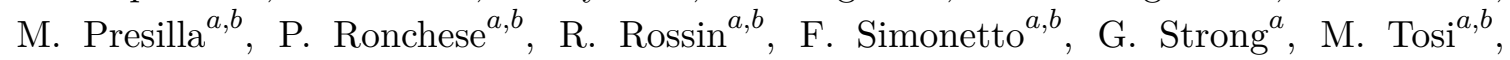
H. YARAR ${ }^{a, b}$, M. Zanetti ${ }^{a, b}$, P. Zotto ${ }^{a, b}$, A. Zucchetta $^{a, b}$, G. Zumerle ${ }^{a, b}$

INFN Sezione di Pavia ${ }^{a}$, Università di Pavia ${ }^{b}$, Pavia, Italy

C. Aime ${ }^{a, b}$, A. Braghieri ${ }^{a}$, S. Calzaferri ${ }^{a, b}$, D. Fiorina ${ }^{a, b}$, P. Montagna ${ }^{a, b}$, S.P. Ratti ${ }^{a, b}$,

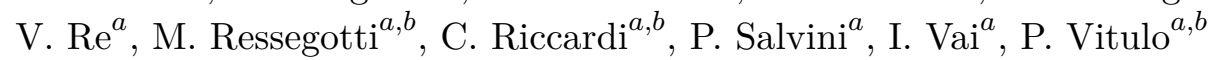


INFN Sezione di Perugia ${ }^{a}$, Università di Perugia ${ }^{b}$, Perugia, Italy G.M. Bilei ${ }^{a}$, D. Ciangottini ${ }^{a, b}$, L. Fanò ${ }^{a, b}$, P. Lariccia ${ }^{a, b}$, M. Magherini $^{b}$, G. Mantovani $^{a, b}{ }^{,}$

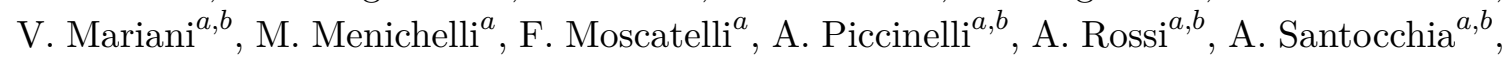
D. Spiga ${ }^{a}$, T. Tedeschi ${ }^{a, b}$

INFN Sezione di Pisa ${ }^{a}$, Università di Pisa ${ }^{b}$, Scuola Normale Superiore di Pisa ${ }^{c}$, Pisa Italy, Università di Siena ${ }^{d}$, Siena, Italy

P. Azzurri $^{a}$, G. Bagliesi ${ }^{a}$, V. Bertacchi ${ }^{a, c}$, L. Bianchini ${ }^{a}$, T. Boccali ${ }^{a}$, E. Bossini ${ }^{a, b}$, R. Castaldi ${ }^{a}$, M.A. Ciocci ${ }^{a, b}$, R. Dell'Orso ${ }^{a}$, M.R. Di Domenico ${ }^{a, d}$, S. Donato $^{a}$, A. Giassi $^{a}$,

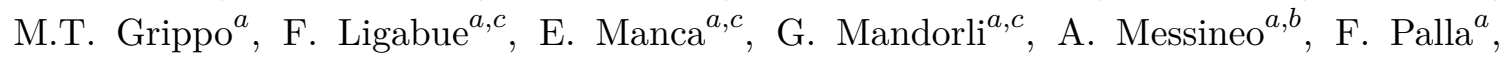
S. Parolia ${ }^{a, b}$, G. Ramirez-Sanchez ${ }^{a, c}$, A. Rizzi ${ }^{a, b}$, G. Rolandi ${ }^{a, c}$, S. Roy Chowdhury ${ }^{a, c}$,

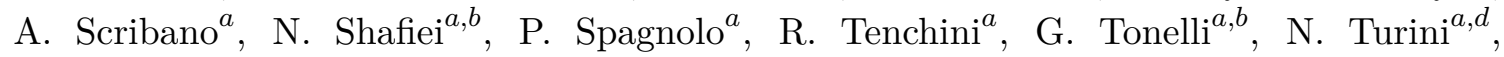
A. Venturi ${ }^{a}$, P.G. Verdini ${ }^{a}$

INFN Sezione di Roma ${ }^{a}$, Sapienza Università di Roma ${ }^{b}$, Rome, Italy

M. Campana ${ }^{a, b}$, F. Cavallari ${ }^{a}$, M. Cipriani ${ }^{a, b}$, D. Del Re ${ }^{a, b}$, E. Di Marco ${ }^{a}$, M. Diemoz $^{a}$, E. Longo $^{a, b}$, P. Meridiani ${ }^{a}$, G. Organtini ${ }^{a, b}$, F. Pandolfi ${ }^{a}$, R. Paramatti ${ }^{a, b}$, C. Quaranta ${ }^{a, b}$,

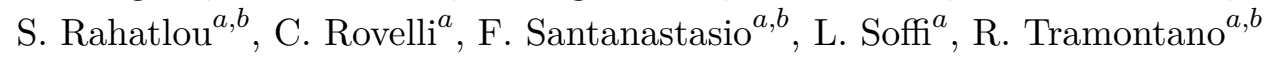

INFN Sezione di Torino ${ }^{a}$, Università di Torino ${ }^{b}$, Torino, Italy, Università del Piemonte Orientale ${ }^{c}$, Novara, Italy

N. Amapane ${ }^{a, b}$, R. Arcidiacono ${ }^{a, c}$, S. Argiro ${ }^{a, b}$, M. Arneodo $^{a, c}$, N. Bartosik ${ }^{a}$, R. Bellan $^{a, b}$,

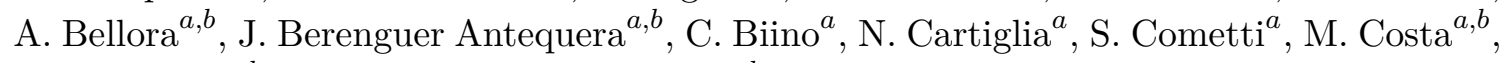
R. Covarelli ${ }^{a, b}$, N. Demaria ${ }^{a}$, B. Kiani ${ }^{a, b}$, F. Legger ${ }^{a}$, C. Mariotti ${ }^{a}$, S. Maselli ${ }^{a}$, E. Migliore $^{a, b}$, E. Monteil ${ }^{a, b}$, M. Monteno ${ }^{a}$, M.M. Obertino ${ }^{a, b}$, G. Ortona $^{a}$, L. Pacher ${ }^{a, b}$, N. Pastrone ${ }^{a}$, M. Pelliccioni ${ }^{a}$, G.L. Pinna Angioni ${ }^{a, b}$, M. Ruspa $^{a, c}$, R. Salvatico ${ }^{a, b}$, K. Shchelina ${ }^{a, b}$, F. Siviero ${ }^{a, b}$, V. Sola $^{a}$, A. Solano ${ }^{a, b}$, D. Soldi ${ }^{a, b}$, A. Staiano $^{a}$, M. Tornago $^{a, b}$, D. Trocino ${ }^{a, b}$, A. Vagnerini

INFN Sezione di Trieste ${ }^{a}$, Università di Trieste ${ }^{b}$, Trieste, Italy

S. Belforte ${ }^{a}$, V. Candelise ${ }^{a, b}$, M. Casarsa ${ }^{a}$, F. Cossutti ${ }^{a}$, A. Da Rold ${ }^{a, b}$, G. Della Ricca ${ }^{a, b}$, G. Sorrentino $^{a, b}$, F. Vazzoler ${ }^{a, b}$

Kyungpook National University, Daegu, Korea

S. Dogra, C. Huh, B. Kim, D.H. Kim, G.N. Kim, J. Kim, J. Lee, S.W. Lee, C.S. Moon, Y.D. Oh, S.I. Pak, B.C. Radburn-Smith, S. Sekmen, Y.C. Yang

Chonnam National University, Institute for Universe and Elementary Particles, Kwangju, Korea

H. Kim, D.H. Moon

Hanyang University, Seoul, Korea

B. Francois, T.J. Kim, J. Park

Korea University, Seoul, Korea

S. Cho, S. Choi, Y. Go, B. Hong, K. Lee, K.S. Lee, J. Lim, J. Park, S.K. Park, J. Yoo 
Kyung Hee University, Department of Physics, Seoul, Republic of Korea

J. Goh, A. Gurtu

Sejong University, Seoul, Korea

H.S. Kim, Y. Kim

Seoul National University, Seoul, Korea

J. Almond, J.H. Bhyun, J. Choi, S. Jeon, J. Kim, J.S. Kim, S. Ko, H. Kwon, H. Lee, S. Lee, B.H. Oh, M. Oh, S.B. Oh, H. Seo, U.K. Yang, I. Yoon

University of Seoul, Seoul, Korea

W. Jang, D. Jeon, D.Y. Kang, Y. Kang, J.H. Kim, S. Kim, B. Ko, J.S.H. Lee, Y. Lee, I.C. Park, Y. Roh, D. Song, I.J. Watson, S. Yang

Yonsei University, Department of Physics, Seoul, Korea

S. Ha, H.D. Yoo

Sungkyunkwan University, Suwon, Korea

Y. Jeong, H. Lee, Y. Lee, I. Yu

College of Engineering and Technology, American University of the Middle East (AUM), Egaila, Kuwait

T. Beyrouthy, Y. Maghrbi

Riga Technical University, Riga, Latvia

V. Veckalns ${ }^{45}$

Vilnius University, Vilnius, Lithuania

M. Ambrozas, A. Juodagalvis, A. Rinkevicius, G. Tamulaitis, A. Vaitkevicius

National Centre for Particle Physics, Universiti Malaya, Kuala Lumpur, Malaysia

N. Bin Norjoharuddeen, W.A.T. Wan Abdullah, M.N. Yusli, Z. Zolkapli

Universidad de Sonora (UNISON), Hermosillo, Mexico

J.F. Benitez, A. Castaneda Hernandez, M. León Coello, J.A. Murillo Quijada, A. Sehrawat, L. Valencia Palomo

Centro de Investigacion y de Estudios Avanzados del IPN, Mexico City, Mexico G. Ayala, H. Castilla-Valdez, I. Heredia-De La Cruz ${ }^{46}$, R. Lopez-Fernandez, C.A. Mondragon Herrera, D.A. Perez Navarro, A. Sanchez-Hernandez

Universidad Iberoamericana, Mexico City, Mexico

S. Carrillo Moreno, C. Oropeza Barrera, M. Ramirez-Garcia, F. Vazquez Valencia

Benemerita Universidad Autonoma de Puebla, Puebla, Mexico

I. Pedraza, H.A. Salazar Ibarguen, C. Uribe Estrada

University of Montenegro, Podgorica, Montenegro

J. Mijuskovic $^{47}$, N. Raicevic 
University of Auckland, Auckland, New Zealand

D. Krofcheck

University of Canterbury, Christchurch, New Zealand

S. Bheesette, P.H. Butler

National Centre for Physics, Quaid-I-Azam University, Islamabad, Pakistan

A. Ahmad, M.I. Asghar, A. Awais, M.I.M. Awan, H.R. Hoorani, W.A. Khan, M.A. Shah, M. Shoaib, M. Waqas

AGH University of Science and Technology Faculty of Computer Science, Electronics and Telecommunications, Krakow, Poland

V. Avati, L. Grzanka, M. Malawski

National Centre for Nuclear Research, Swierk, Poland

H. Bialkowska, M. Bluj, B. Boimska, M. Górski, M. Kazana, M. Szleper, P. Zalewski

Institute of Experimental Physics, Faculty of Physics, University of Warsaw, Warsaw, Poland

K. Bunkowski, K. Doroba, A. Kalinowski, M. Konecki, J. Krolikowski, M. Walczak

Laboratório de Instrumentação e Física Experimental de Partículas, Lisboa, Portugal

M. Araujo, P. Bargassa, D. Bastos, A. Boletti, P. Faccioli, M. Gallinaro, J. Hollar, N. Leonardo, T. Niknejad, M. Pisano, J. Seixas, O. Toldaiev, J. Varela

Joint Institute for Nuclear Research, Dubna, Russia

S. Afanasiev, D. Budkouski, I. Golutvin, I. Gorbunov, V. Karjavine, V. Korenkov, A. Lanev, A. Malakhov, V. Matveev ${ }^{48,49}$, V. Palichik, V. Perelygin, M. Savina, D. Seitova, V. Shalaev, S. Shmatov, S. Shulha, V. Smirnov, O. Teryaev, N. Voytishin, B.S. Yuldashev ${ }^{50}$, A. Zarubin, I. Zhizhin

Petersburg Nuclear Physics Institute, Gatchina (St. Petersburg), Russia

G. Gavrilov, V. Golovtcov, Y. Ivanov, V. Kim ${ }^{51}$, E. Kuznetsova ${ }^{52}$, V. Murzin, V. Oreshkin, I. Smirnov, D. Sosnov, V. Sulimov, L. Uvarov, S. Volkov, A. Vorobyev

Institute for Nuclear Research, Moscow, Russia

Yu. Andreev, A. Dermenev, S. Gninenko, N. Golubev, A. Karneyeu, D. Kirpichnikov, M. Kirsanov, N. Krasnikov, A. Pashenkov, G. Pivovarov, D. Tlisov ${ }^{\dagger}$, A. Toropin

Institute for Theoretical and Experimental Physics named by A.I. Alikhanov of NRC 'Kurchatov Institute', Moscow, Russia

V. Epshteyn, V. Gavrilov, N. Lychkovskaya, A. Nikitenko ${ }^{53}$, V. Popov, A. Spiridonov, A. Stepennov, M. Toms, E. Vlasov, A. Zhokin

Moscow Institute of Physics and Technology, Moscow, Russia

T. Aushev 
National Research Nuclear University 'Moscow Engineering Physics Institute' (MEPhI), Moscow, Russia

M. Chadeeva ${ }^{54}$, A. Oskin, P. Parygin, E. Popova, V. Rusinov

P.N. Lebedev Physical Institute, Moscow, Russia

V. Andreev, M. Azarkin, I. Dremin, M. Kirakosyan, A. Terkulov

Skobeltsyn Institute of Nuclear Physics, Lomonosov Moscow State University, Moscow, Russia

A. Belyaev, E. Boos, M. Dubinin ${ }^{55}$, L. Dudko, A. Ershov, V. Klyukhin, O. Kodolova, I. Lokhtin, O. Lukina, S. Obraztsov, S. Petrushanko, V. Savrin, A. Snigirev

Novosibirsk State University (NSU), Novosibirsk, Russia

V. Blinov ${ }^{56}$, T. Dimova ${ }^{56}$, L. Kardapoltsev ${ }^{56}$, A. Kozyrev ${ }^{56}$, I. Ovtin ${ }^{56}$, Y. Skovpen ${ }^{56}$

Institute for High Energy Physics of National Research Centre 'Kurchatov Institute', Protvino, Russia

I. Azhgirey, I. Bayshev, D. Elumakhov, V. Kachanov, D. Konstantinov, P. Mandrik, V. Petrov, R. Ryutin, S. Slabospitskii, A. Sobol, S. Troshin, N. Tyurin, A. Uzunian, A. Volkov

National Research Tomsk Polytechnic University, Tomsk, Russia

A. Babaev, V. Okhotnikov

Tomsk State University, Tomsk, Russia

V. Borchsh, V. Ivanchenko, E. Tcherniaev

University of Belgrade: Faculty of Physics and VINCA Institute of Nuclear Sciences, Belgrade, Serbia

P. Adzic ${ }^{57}$, M. Dordevic, P. Milenovic, J. Milosevic

Centro de Investigaciones Energéticas Medioambientales y Tecnológicas (CIEMAT), Madrid, Spain

M. Aguilar-Benitez, J. Alcaraz Maestre, A. Álvarez Fernández, I. Bachiller, M. Barrio Luna, Cristina F. Bedoya, C.A. Carrillo Montoya, M. Cepeda, M. Cerrada, N. Colino, B. De La Cruz, A. Delgado Peris, J.P. Fernández Ramos, J. Flix, M.C. Fouz, O. Gonzalez Lopez, S. Goy Lopez, J.M. Hernandez, M.I. Josa, J. León Holgado, D. Moran, Á. Navarro Tobar, A. Pérez-Calero Yzquierdo, J. Puerta Pelayo, I. Redondo, L. Romero, S. Sánchez Navas, L. Urda Gómez, C. Willmott

Universidad Autónoma de Madrid, Madrid, Spain

J.F. de Trocóniz, R. Reyes-Almanza

Universidad de Oviedo, Instituto Universitario de Ciencias y Tecnologías Espaciales de Asturias (ICTEA), Oviedo, Spain

B. Alvarez Gonzalez, J. Cuevas, C. Erice, J. Fernandez Menendez, S. Folgueras, I. Gonzalez Caballero, E. Palencia Cortezon, C. Ramón Álvarez, J. Ripoll Sau, V. Rodríguez Bouza, A. Trapote, N. Trevisani 
Instituto de Física de Cantabria (IFCA), CSIC-Universidad de Cantabria, Santander, Spain

J.A. Brochero Cifuentes, I.J. Cabrillo, A. Calderon, B. Chazin Quero, J. Duarte Campderros, M. Fernandez, C. Fernandez Madrazo, P.J. Fernández Manteca, A. García Alonso, G. Gomez, C. Martinez Rivero, P. Martinez Ruiz del Arbol, F. Matorras, P. Matorras Cuevas, J. Piedra Gomez, C. Prieels, T. Rodrigo, A. Ruiz-Jimeno, L. Scodellaro, I. Vila, J.M. Vizan Garcia

\section{University of Colombo, Colombo, Sri Lanka}

M.K. Jayananda, B. Kailasapathy ${ }^{58}$, D.U.J. Sonnadara, D.D.C. Wickramarathna

\section{University of Ruhuna, Department of Physics, Matara, Sri Lanka}

W.G.D. Dharmaratna, K. Liyanage, N. Perera, N. Wickramage

\section{CERN, European Organization for Nuclear Research, Geneva, Switzerland}

T.K. Aarrestad, D. Abbaneo, J. Alimena, E. Auffray, G. Auzinger, J. Baechler, P. Baillon ${ }^{\dagger}$, D. Barney, J. Bendavid, M. Bianco, A. Bocci, T. Camporesi, M. Capeans Garrido, G. Cerminara, S.S. Chhibra, L. Cristella, D. d'Enterria, A. Dabrowski, N. Daci, A. David, A. De Roeck, M.M. Defranchis, M. Deile, M. Dobson, M. Dünser, N. Dupont, A. ElliottPeisert, N. Emriskova, F. Fallavollita ${ }^{59}$, D. Fasanella, S. Fiorendi, A. Florent, G. Franzoni, W. Funk, S. Giani, D. Gigi, K. Gill, F. Glege, L. Gouskos, M. Haranko, J. Hegeman, Y. Iiyama, V. Innocente, T. James, P. Janot, J. Kaspar, J. Kieseler, M. Komm, N. Kratochwil, C. Lange, S. Laurila, P. Lecoq, K. Long, C. Lourenço, L. Malgeri, S. Mallios, M. Mannelli, A.C. Marini, F. Meijers, S. Mersi, E. Meschi, F. Moortgat, M. Mulders, S. Orfanelli, L. Orsini, F. Pantaleo, L. Pape, E. Perez, M. Peruzzi, A. Petrilli, G. Petrucciani, A. Pfeiffer, M. Pierini, D. Piparo, M. Pitt, H. Qu, T. Quast, D. Rabady, A. Racz, G. Reales Gutiérrez, M. Rieger, M. Rovere, H. Sakulin, J. Salfeld-Nebgen, S. Scarfi, C. Schäfer, C. Schwick, M. Selvaggi, A. Sharma, P. Silva, W. Snoeys, P. Sphicas ${ }^{60}$, S. Summers, V.R. Tavolaro, D. Treille, A. Tsirou, G.P. Van Onsem, M. Verzetti, J. Wanczyk ${ }^{61}$, K.A. Wozniak, W.D. Zeuner

\section{Paul Scherrer Institut, Villigen, Switzerland}

L. Caminada ${ }^{62}$, A. Ebrahimi, W. Erdmann, R. Horisberger, Q. Ingram, H.C. Kaestli, D. Kotlinski, U. Langenegger, M. Missiroli, T. Rohe

ETH Zurich - Institute for Particle Physics and Astrophysics (IPA), Zurich, Switzerland

K. Androsov ${ }^{61}$, M. Backhaus, P. Berger, A. Calandri, N. Chernyavskaya, A. De Cosa, G. Dissertori, M. Dittmar, M. Donegà, C. Dorfer, F. Eble, T.A. Gómez Espinosa, C. Grab, D. Hits, W. Lustermann, A.-M. Lyon, R.A. Manzoni, C. Martin Perez, M.T. Meinhard, F. Micheli, F. Nessi-Tedaldi, J. Niedziela, F. Pauss, V. Perovic, G. Perrin, S. Pigazzini, M.G. Ratti, M. Reichmann, C. Reissel, T. Reitenspiess, B. Ristic, D. Ruini, D.A. Sanz Becerra, M. Schönenberger, V. Stampf, J. Steggemann ${ }^{61}$, R. Wallny, D.H. Zhu 
Universität Zürich, Zurich, Switzerland

C. Amsler ${ }^{63}$, P. Bärtschi, C. Botta, D. Brzhechko, M.F. Canelli, K. Cormier, A. De Wit,

R. Del Burgo, J.K. Heikkilä, M. Huwiler, A. Jofrehei, B. Kilminster, S. Leontsinis,

A. Macchiolo, P. Meiring, V.M. Mikuni, U. Molinatti, I. Neutelings, G. Rauco, A. Reimers,

P. Robmann, S. Sanchez Cruz, K. Schweiger, Y. Takahashi

National Central University, Chung-Li, Taiwan

C. Adloff ${ }^{64}$, C.M. Kuo, W. Lin, A. Roy, T. Sarkar ${ }^{36}$, S.S. Yu

National Taiwan University (NTU), Taipei, Taiwan

L. Ceard, Y. Chao, K.F. Chen, P.H. Chen, W.-S. Hou, Y.y. Li, R.-S. Lu, E. Paganis,

A. Psallidas, A. Steen, H.y. Wu, E. Yazgan, P.r. Yu

Chulalongkorn University, Faculty of Science, Department of Physics, Bangkok, Thailand

B. Asavapibhop, C. Asawatangtrakuldee, N. Srimanobhas

Çukurova University, Physics Department, Science and Art Faculty, Adana, Turkey

F. Boran, S. Damarseckin ${ }^{65}$, Z.S. Demiroglu, F. Dolek, I. Dumanoglu ${ }^{66}$, E. Eskut, Y. Guler,

E. Gurpinar Guler ${ }^{67}$, I. Hos $^{68}$, C. Isik, O. Kara, A. Kayis Topaksu, U. Kiminsu, G. Onengut,

K. Ozdemir ${ }^{69}$, A. Polatoz, A.E. Simsek, B. Tali ${ }^{70}$, U.G. Tok, S. Turkcapar, I.S. Zorbakir,

C. Zorbilmez

Middle East Technical University, Physics Department, Ankara, Turkey

B. Isildak ${ }^{71}$, G. Karapinar ${ }^{72}$, K. Ocalan ${ }^{73}$, M. Yalvac ${ }^{74}$

Bogazici University, Istanbul, Turkey

B. Akgun, I.O. Atakisi, E. Gülmez, M. Kaya ${ }^{75}$, O. Kaya ${ }^{76}$, Ö. Özçelik, S. Tekten ${ }^{77}$, E.A. Yetkin ${ }^{78}$

Istanbul Technical University, Istanbul, Turkey

A. Cakir, K. Cankocak ${ }^{66}$, Y. Komurcu, S. Sen ${ }^{79}$

Istanbul University, Istanbul, Turkey

S. Cerci ${ }^{70}$, B. Kaynak, S. Ozkorucuklu, D. Sunar Cerci ${ }^{70}$

Institute for Scintillation Materials of National Academy of Science of Ukraine, Kharkov, Ukraine

B. Grynyov

National Scientific Center, Kharkov Institute of Physics and Technology, Kharkov, Ukraine

L. Levchuk

University of Bristol, Bristol, United Kingdom

D. Anthony, E. Bhal, S. Bologna, J.J. Brooke, A. Bundock, E. Clement, D. Cussans,

H. Flacher, J. Goldstein, G.P. Heath, H.F. Heath, L. Kreczko, B. Krikler, S. Paramesvaran,

S. Seif El Nasr-Storey, V.J. Smith, N. Stylianou ${ }^{80}$, R. White 


\section{Rutherford Appleton Laboratory, Didcot, United Kingdom}

K.W. Bell, A. Belyaev ${ }^{81}$, C. Brew, R.M. Brown, D.J.A. Cockerill, K.V. Ellis, K. Harder, S. Harper, J. Linacre, K. Manolopoulos, D.M. Newbold, E. Olaiya, D. Petyt, T. Reis, T. Schuh, C.H. Shepherd-Themistocleous, I.R. Tomalin, T. Williams

\section{Imperial College, London, United Kingdom}

R. Bainbridge, P. Bloch, S. Bonomally, J. Borg, S. Breeze, O. Buchmuller, V. Cepaitis, G.S. Chahal ${ }^{82}$, D. Colling, P. Dauncey, G. Davies, M. Della Negra, S. Fayer, G. Fedi, G. Hall, M.H. Hassanshahi, G. Iles, J. Langford, L. Lyons, A.-M. Magnan, S. Malik, A. Martelli, J. Nash ${ }^{83}$, M. Pesaresi, D.M. Raymond, A. Richards, A. Rose, E. Scott, C. Seez, A. Shtipliyski, A. Tapper, K. Uchida, T. Virdee ${ }^{19}$, N. Wardle, S.N. Webb, D. Winterbottom, A.G. Zecchinelli

\section{Brunel University, Uxbridge, United Kingdom}

K. Coldham, J.E. Cole, A. Khan, P. Kyberd, I.D. Reid, L. Teodorescu, S. Zahid

Baylor University, Waco, U.S.A.

S. Abdullin, A. Brinkerhoff, B. Caraway, J. Dittmann, K. Hatakeyama, A.R. Kanuganti, B. McMaster, N. Pastika, S. Sawant, C. Sutantawibul, J. Wilson

Catholic University of America, Washington, DC, U.S.A.

R. Bartek, A. Dominguez, R. Uniyal, A.M. Vargas Hernandez

The University of Alabama, Tuscaloosa, U.S.A.

A. Buccilli, S.I. Cooper, D. Di Croce, S.V. Gleyzer, C. Henderson, C.U. Perez, P. Rumerio ${ }^{84}$, C. West

Boston University, Boston, U.S.A.

A. Akpinar, A. Albert, D. Arcaro, C. Cosby, Z. Demiragli, E. Fontanesi, D. Gastler, J. Rohlf, K. Salyer, D. Sperka, D. Spitzbart, I. Suarez, A. Tsatsos, S. Yuan, D. Zou

Brown University, Providence, U.S.A.

G. Benelli, B. Burkle, X. Coubez ${ }^{20}$, D. Cutts, Y.t. Duh, M. Hadley, U. Heintz, J.M. $\operatorname{Hogan}^{85}$, G. Landsberg, K.T. Lau, J. Lee, M. Lukasik, J. Luo, M. Narain, S. Sagir ${ }^{86}$, E. Usai, W.Y. Wong, X. Yan, D. Yu, W. Zhang

University of California, Davis, Davis, U.S.A.

J. Bonilla, C. Brainerd, R. Breedon, M. Calderon De La Barca Sanchez, M. Chertok, J. Conway, P.T. Cox, R. Erbacher, G. Haza, F. Jensen, O. Kukral, R. Lander, M. Mulhearn, D. Pellett, B. Regnery, D. Taylor, Y. Yao, F. Zhang

\section{University of California, Los Angeles, U.S.A.}

M. Bachtis, R. Cousins, A. Datta, D. Hamilton, J. Hauser, M. Ignatenko, M.A. Iqbal, T. Lam, N. Mccoll, W.A. Nash, S. Regnard, D. Saltzberg, B. Stone, V. Valuev

University of California, Riverside, Riverside, U.S.A.

K. Burt, Y. Chen, R. Clare, J.W. Gary, M. Gordon, G. Hanson, G. Karapostoli, O.R. Long, N. Manganelli, M. Olmedo Negrete, W. Si, S. Wimpenny, Y. Zhang 
University of California, San Diego, La Jolla, U.S.A.

J.G. Branson, P. Chang, S. Cittolin, S. Cooperstein, N. Deelen, J. Duarte, R. Gerosa, L. Giannini, D. Gilbert, J. Guiang, R. Kansal, V. Krutelyov, R. Lee, J. Letts, M. Masciovecchio, S. May, M. Pieri, B.V. Sathia Narayanan, V. Sharma, M. Tadel, A. Vartak, F. Würthwein, Y. Xiang, A. Yagil

University of California, Santa Barbara - Department of Physics, Santa Barbara, U.S.A.

N. Amin, C. Campagnari, M. Citron, A. Dorsett, V. Dutta, J. Incandela, M. Kilpatrick, J. Kim, B. Marsh, H. Mei, M. Oshiro, M. Quinnan, J. Richman, U. Sarica, D. Stuart, S. Wang

California Institute of Technology, Pasadena, U.S.A.

A. Bornheim, O. Cerri, I. Dutta, J.M. Lawhorn, N. Lu, J. Mao, H.B. Newman, J. Ngadiuba, T.Q. Nguyen, M. Spiropulu, J.R. Vlimant, C. Wang, S. Xie, Z. Zhang, R.Y. Zhu

Carnegie Mellon University, Pittsburgh, U.S.A.

J. Alison, S. An, M.B. Andrews, P. Bryant, T. Ferguson, A. Harilal, T. Mudholkar, M. Paulini, A. Sanchez

University of Colorado Boulder, Boulder, U.S.A.

J.P. Cumalat, W.T. Ford, E. MacDonald, R. Patel, A. Perloff, K. Stenson, K.A. Ulmer, S.R. Wagner

Cornell University, Ithaca, U.S.A.

J. Alexander, Y. Cheng, J. Chu, D.J. Cranshaw, K. Mcdermott, J. Monroy, J.R. Patterson, D. Quach, J. Reichert, A. Ryd, W. Sun, S.M. Tan, Z. Tao, J. Thom, P. Wittich, M. Zientek

Fermi National Accelerator Laboratory, Batavia, U.S.A.

M. Albrow, M. Alyari, G. Apollinari, A. Apresyan, A. Apyan, S. Banerjee, L.A.T. Bauerdick, D. Berry, J. Berryhill, P.C. Bhat, K. Burkett, J.N. Butler, A. Canepa, G.B. Cerati, H.W.K. Cheung, F. Chlebana, M. Cremonesi, K.F. Di Petrillo, V.D. Elvira, Y. Feng, J. Freeman, Z. Gecse, L. Gray, D. Green, S. Grünendahl, O. Gutsche, R.M. Harris, R. Heller, T.C. Herwig, J. Hirschauer, B. Jayatilaka, S. Jindariani, M. Johnson, U. Joshi, T. Klijnsma, B. Klima, K.H.M. Kwok, S. Lammel, D. Lincoln, R. Lipton, T. Liu, C. Madrid, K. Maeshima, C. Mantilla, D. Mason, P. McBride, P. Merkel, S. Mrenna, S. Nahn, V. O'Dell, V. Papadimitriou, K. Pedro, C. Pena ${ }^{55}$, O. Prokofyev, F. Ravera, A. Reinsvold Hall, L. Ristori, B. Schneider, E. Sexton-Kennedy, N. Smith, A. Soha, W.J. Spalding, L. Spiegel, S. Stoynev, J. Strait, L. Taylor, S. Tkaczyk, N.V. Tran, L. Uplegger, E.W. Vaandering, H.A. Weber

\section{University of Florida, Gainesville, U.S.A.}

D. Acosta, P. Avery, D. Bourilkov, L. Cadamuro, V. Cherepanov, F. Errico, R.D. Field, D. Guerrero, B.M. Joshi, M. Kim, E. Koenig, J. Konigsberg, A. Korytov, K.H. Lo, K. Matchev, N. Menendez, G. Mitselmakher, A. Muthirakalayil Madhu, N. Rawal, D. Rosenzweig, S. Rosenzweig, K. Shi, J. Sturdy, J. Wang, E. Yigitbasi, X. Zuo 
Florida State University, Tallahassee, U.S.A.

T. Adams, A. Askew, D. Diaz, R. Habibullah, V. Hagopian, K.F. Johnson, R. Khurana,

T. Kolberg, G. Martinez, H. Prosper, C. Schiber, R. Yohay, J. Zhang

Florida Institute of Technology, Melbourne, U.S.A.

M.M. Baarmand, S. Butalla, T. Elkafrawy ${ }^{14}$, M. Hohlmann, R. Kumar Verma, D. Noonan, M. Rahmani, M. Saunders, F. Yumiceva

University of Illinois at Chicago (UIC), Chicago, U.S.A.

M.R. Adams, H. Becerril Gonzalez, R. Cavanaugh, X. Chen, S. Dittmer, O. Evdokimov, C.E. Gerber, D.A. Hangal, D.J. Hofman, A.H. Merrit, C. Mills, G. Oh, T. Roy, S. Rudrabhatla, M.B. Tonjes, N. Varelas, J. Viinikainen, X. Wang, Z. Wu, Z. Ye

The University of Iowa, Iowa City, U.S.A.

M. Alhusseini, K. Dilsiz ${ }^{87}$, R.P. Gandrajula, O.K. Köseyan, J.-P. Merlo, A. Mestvirishvili ${ }^{88}$, J. Nachtman, H. Ogul ${ }^{89}$, Y. Onel, A. Penzo, C. Snyder, E. Tiras ${ }^{90}$

Johns Hopkins University, Baltimore, U.S.A.

O. Amram, B. Blumenfeld, L. Corcodilos, J. Davis, M. Eminizer, A.V. Gritsan, S. Kyriacou, P. Maksimovic, J. Roskes, M. Swartz, T.Á. Vámi

The University of Kansas, Lawrence, U.S.A.

J. Anguiano, C. Baldenegro Barrera, P. Baringer, A. Bean, A. Bylinkin, T. Isidori, S. Khalil, J. King, G. Krintiras, A. Kropivnitskaya, C. Lindsey, N. Minafra, M. Murray, C. Rogan, C. Royon, S. Sanders, E. Schmitz, C. Smith, J.D. Tapia Takaki, Q. Wang, J. Williams, G. Wilson

\section{Kansas State University, Manhattan, U.S.A.}

S. Duric, A. Ivanov, K. Kaadze, D. Kim, Y. Maravin, T. Mitchell, A. Modak, K. Nam

Lawrence Livermore National Laboratory, Livermore, U.S.A.

F. Rebassoo, D. Wright

University of Maryland, College Park, U.S.A.

E. Adams, A. Baden, O. Baron, A. Belloni, S.C. Eno, N.J. Hadley, S. Jabeen, R.G. Kellogg,

T. Koeth, A.C. Mignerey, S. Nabili, M. Seidel, A. Skuja, L. Wang, K. Wong

Massachusetts Institute of Technology, Cambridge, U.S.A.

D. Abercrombie, G. Andreassi, R. Bi, S. Brandt, W. Busza, I.A. Cali, Y. Chen, M. D'Alfonso, J. Eysermans, G. Gomez Ceballos, M. Goncharov, P. Harris, M. Hu, M. Klute, D. Kovalskyi, J. Krupa, Y.-J. Lee, B. Maier, C. Mironov, C. Paus, D. Rankin, C. Roland, G. Roland, Z. Shi, G.S.F. Stephans, K. Tatar, J. Wang, Z. Wang, B. Wyslouch

University of Minnesota, Minneapolis, U.S.A.

R.M. Chatterjee, A. Evans, P. Hansen, J. Hiltbrand, Sh. Jain, M. Krohn, Y. Kubota, J. Mans, M. Revering, R. Rusack, R. Saradhy, N. Schroeder, N. Strobbe, M.A. Wadud 
University of Nebraska-Lincoln, Lincoln, U.S.A.

K. Bloom, M. Bryson, S. Chauhan, D.R. Claes, C. Fangmeier, L. Finco, F. Golf, J.R. González Fernández, C. Joo, I. Kravchenko, M. Musich, I. Reed, J.E. Siado, G.R. Snow ${ }^{\dagger}$, W. Tabb, F. Yan

State University of New York at Buffalo, Buffalo, U.S.A.

G. Agarwal, H. Bandyopadhyay, L. Hay, I. Iashvili, A. Kharchilava, C. McLean, D. Nguyen, J. Pekkanen, S. Rappoccio, A. Williams

Northeastern University, Boston, U.S.A.

G. Alverson, E. Barberis, C. Freer, Y. Haddad, A. Hortiangtham, J. Li, G. Madigan, B. Marzocchi, D.M. Morse, V. Nguyen, T. Orimoto, A. Parker, L. Skinnari, A. TishelmanCharny, T. Wamorkar, B. Wang, A. Wisecarver, D. Wood

Northwestern University, Evanston, U.S.A.

S. Bhattacharya, J. Bueghly, Z. Chen, A. Gilbert, T. Gunter, K.A. Hahn, N. Odell, M.H. Schmitt, M. Velasco

University of Notre Dame, Notre Dame, U.S.A.

R. Band, R. Bucci, A. Das, N. Dev, R. Goldouzian, M. Hildreth, K. Hurtado Anampa, C. Jessop, K. Lannon, N. Loukas, N. Marinelli, I. Mcalister, T. McCauley, F. Meng, K. Mohrman, Y. Musienko ${ }^{48}$, R. Ruchti, P. Siddireddy, M. Wayne, A. Wightman, M. Wolf, M. Zarucki, L. Zygala

The Ohio State University, Columbus, U.S.A.

B. Bylsma, B. Cardwell, L.S. Durkin, B. Francis, C. Hill, M. Nunez Ornelas, K. Wei, B.L. Winer, B.R. Yates

Princeton University, Princeton, U.S.A.

F.M. Addesa, B. Bonham, P. Das, G. Dezoort, P. Elmer, A. Frankenthal, B. Greenberg, N. Haubrich, S. Higginbotham, A. Kalogeropoulos, G. Kopp, S. Kwan, D. Lange, M.T. Lucchini, D. Marlow, K. Mei, I. Ojalvo, J. Olsen, C. Palmer, D. Stickland, C. Tully

University of Puerto Rico, Mayaguez, U.S.A.

S. Malik, S. Norberg

Purdue University, West Lafayette, U.S.A.

A.S. Bakshi, V.E. Barnes, R. Chawla, S. Das, L. Gutay, M. Jones, A.W. Jung, S. Karmarkar, M. Liu, G. Negro, N. Neumeister, G. Paspalaki, C.C. Peng, S. Piperov, A. Purohit, J.F. Schulte, M. Stojanovic ${ }^{15}$, J. Thieman, F. Wang, R. Xiao, W. Xie

Purdue University Northwest, Hammond, U.S.A.

J. Dolen, N. Parashar

Rice University, Houston, U.S.A.

A. Baty, M. Decaro, S. Dildick, K.M. Ecklund, S. Freed, P. Gardner, F.J.M. Geurts, A. Kumar, W. Li, B.P. Padley, R. Redjimi, W. Shi, A.G. Stahl Leiton, S. Yang, L. Zhang, Y. Zhang 
University of Rochester, Rochester, U.S.A.

A. Bodek, P. de Barbaro, R. Demina, J.L. Dulemba, C. Fallon, T. Ferbel, M. Galanti, A. Garcia-Bellido, O. Hindrichs, A. Khukhunaishvili, E. Ranken, R. Taus

Rutgers, The State University of New Jersey, Piscataway, U.S.A.

B. Chiarito, J.P. Chou, A. Gandrakota, Y. Gershtein, E. Halkiadakis, A. Hart, M. Heindl, E. Hughes, S. Kaplan, O. Karacheban ${ }^{23}$, I. Laflotte, A. Lath, R. Montalvo, K. Nash, M. Osherson, S. Salur, S. Schnetzer, S. Somalwar, R. Stone, S.A. Thayil, S. Thomas, H. Wang

University of Tennessee, Knoxville, U.S.A.

H. Acharya, A.G. Delannoy, S. Spanier

Texas A\&M University, College Station, U.S.A.

O. Bouhali ${ }^{91}$, M. Dalchenko, A. Delgado, R. Eusebi, J. Gilmore, T. Huang, T. Kamon ${ }^{92}$, H. Kim, S. Luo, S. Malhotra, R. Mueller, D. Overton, D. Rathjens, A. Safonov

Texas Tech University, Lubbock, U.S.A.

N. Akchurin, J. Damgov, V. Hegde, S. Kunori, K. Lamichhane, S.W. Lee, T. Mengke, S. Muthumuni, T. Peltola, I. Volobouev, Z. Wang, A. Whitbeck

Vanderbilt University, Nashville, U.S.A.

E. Appelt, S. Greene, A. Gurrola, W. Johns, A. Melo, H. Ni, K. Padeken, F. Romeo, P. Sheldon, S. Tuo, J. Velkovska

University of Virginia, Charlottesville, U.S.A.

M.W. Arenton, B. Cox, G. Cummings, J. Hakala, R. Hirosky, M. Joyce, A. Ledovskoy, A. Li, C. Neu, B. Tannenwald, S. White, E. Wolfe

Wayne State University, Detroit, U.S.A.

N. Poudyal, P. Thapa

University of Wisconsin - Madison, Madison, WI, U.S.A.

K. Black, T. Bose, J. Buchanan, C. Caillol, S. Dasu, I. De Bruyn, P. Everaerts, F. Fienga, C. Galloni, H. He, M. Herndon, A. Hervé, U. Hussain, A. Lanaro, A. Loeliger, R. Loveless, J. Madhusudanan Sreekala, A. Mallampalli, A. Mohammadi, D. Pinna, A. Savin, V. Shang, V. Sharma, W.H. Smith, D. Teague, S. Trembath-Reichert, W. Vetens

$\dagger$ : Deceased

1: Also at TU Wien, Wien, Austria

2: Also at Institute of Basic and Applied Sciences, Faculty of Engineering, Arab Academy for Science, Technology and Maritime Transport, Alexandria, Egypt, Alexandria, Egypt

3: Also at Université Libre de Bruxelles, Bruxelles, Belgium

4: Also at Universidade Estadual de Campinas, Campinas, Brazil

5: Also at Federal University of Rio Grande do Sul, Porto Alegre, Brazil

6: Also at University of Chinese Academy of Sciences, Beijing, China

7: Also at Department of Physics, Tsinghua University, Beijing, China, Beijing, China

8: Also at UFMS, Nova Andradina, Brazil

9: Also at Nanjing Normal University Department of Physics, Nanjing, China 
10: Now at The University of Iowa, Iowa City, U.S.A:

11: Also at Institute for Theoretical and Experimental Physics named by A.I. Alikhanov of NRC 'Kurchatov Institute', Moscow, Russia

12: Also at Joint Institute for Nuclear Research, Dubna, Russia

13: Also at Cairo University, Cairo, Egypt

14: Also at Ain Shams University, Cairo, Egypt

15: Also at Purdue University, West Lafayette, U.S.A:

16: Also at Université de Haute Alsace, Mulhouse, France

17: Also at Tbilisi State University, Tbilisi, Georgia

18: Also at Erzincan Binali Yildirim University, Erzincan, Turkey

19: Also at CERN, European Organization for Nuclear Research, Geneva, Switzerland

20: Also at RWTH Aachen University, III. Physikalisches Institut A, Aachen, Germany

21: Also at University of Hamburg, Hamburg, Germany

22: Also at Department of Physics, Isfahan University of Technology, Isfahan, Iran, Isfahan, Iran

23: Also at Brandenburg University of Technology, Cottbus, Germany

24: Also at Skobeltsyn Institute of Nuclear Physics, Lomonosov Moscow State University, Moscow, Russia

25: Also at Physics Department, Faculty of Science, Assiut University, Assiut, Egypt

26: Also at Karoly Robert Campus, MATE Institute of Technology, Gyongyos, Hungary

27: Also at Institute of Physics, University of Debrecen, Debrecen, Hungary, Debrecen, Hungary

28: Also at Institute of Nuclear Research ATOMKI, Debrecen, Hungary

29: Also at MTA-ELTE Lendület CMS Particle and Nuclear Physics Group, Eötvös Loránd University, Budapest, Hungary, Budapest, Hungary

30: Also at Wigner Research Centre for Physics, Budapest, Hungary

31: Also at IIT Bhubaneswar, Bhubaneswar, India, Bhubaneswar, India

32: Also at Institute of Physics, Bhubaneswar, India

33: Also at G.H.G. Khalsa College, Punjab, India

34: Also at Shoolini University, Solan, India

35: Also at University of Hyderabad, Hyderabad, India

36: Also at University of Visva-Bharati, Santiniketan, India

37: Also at Indian Institute of Technology (IIT), Mumbai, India

38: Also at Deutsches Elektronen-Synchrotron, Hamburg, Germany

39: Also at Sharif University of Technology, Tehran, Iran

40: Also at Department of Physics, University of Science and Technology of Mazandaran, Behshahr, Iran

41: Now at INFN Sezione di Bari, Università di Bari, and Politecnico di Bari, Bari, Italy

42: Also at Italian National Agency for New Technologies, Energy and Sustainable Economic Development, Bologna, Italy

43: Also at Centro Siciliano di Fisica Nucleare e di Struttura Della Materia, Catania, Italy

44: Also at Università di Napoli 'Federico II', NAPOLI, Italy

45: Also at Riga Technical University, Riga, Latvia, Riga, Latvia

46: Also at Consejo Nacional de Ciencia y Tecnología, Mexico City, Mexico

47: Also at IRFU, CEA, Université Paris-Saclay, Gif-sur-Yvette, France

48: Also at Institute for Nuclear Research, Moscow, Russia

49: Now at National Research Nuclear University 'Moscow Engineering Physics Institute' (MEPhI), Moscow, Russia

50: Also at Institute of Nuclear Physics of the Uzbekistan Academy of Sciences, Tashkent, Uzbekistan 
51: Also at St. Petersburg State Polytechnical University, St. Petersburg, Russia

52: Also at University of Florida, Gainesville, U.S.A:

53: Also at Imperial College, London, United Kingdom

54: Also at P.N. Lebedev Physical Institute, Moscow, Russia

55: Also at California Institute of Technology, Pasadena, U.S.A:

56: Also at Budker Institute of Nuclear Physics, Novosibirsk, Russia

57: Also at Faculty of Physics, University of Belgrade, Belgrade, Serbia

58: Also at Trincomalee Campus, Eastern University, Sri Lanka, Nilaveli, Sri Lanka

59: Also at INFN Sezione di Pavia and Università di Pavia, Pavia, Italy, Pavia, Italy

60: Also at National and Kapodistrian University of Athens, Athens, Greece

61: Also at Ecole Polytechnique Fédérale Lausanne, Lausanne, Switzerland

62: Also at Universität Zürich, Zurich, Switzerland

63: Also at Stefan Meyer Institute for Subatomic Physics, Vienna, Austria, Vienna, Austria

64: Also at Laboratoire d'Annecy-le-Vieux de Physique des Particules, IN2P3-CNRS, Annecyle-Vieux, France

65: Also at Şrnak University, Sirnak, Turkey

66: Also at Near East University, Research Center of Experimental Health Science, Nicosia, Turkey

67: Also at Konya Technical University, Konya, Turkey

68: Also at Istanbul University - Cerrahpasa, Faculty of Engineering, Istanbul, Turkey

69: Also at Piri Reis University, Istanbul, Turkey

70: Also at Adiyaman University, Adiyaman, Turkey

71: Also at Ozyegin University, Istanbul, Turkey

72: Also at Izmir Institute of Technology, Izmir, Turkey

73: Also at Necmettin Erbakan University, Konya, Turkey

74: Also at Bozok Universitetesi Rektörlügü, Yozgat, Turkey, Yozgat, Turkey

75: Also at Marmara University, Istanbul, Turkey

76: Also at Milli Savunma University, Istanbul, Turkey

77: Also at Kafkas University, Kars, Turkey

78: Also at Istanbul Bilgi University, Istanbul, Turkey

79: Also at Hacettepe University, Ankara, Turkey

80: Also at Vrije Universiteit Brussel, Brussel, Belgium

81: Also at School of Physics and Astronomy, University of Southampton, Southampton, United Kingdom

82: Also at IPPP Durham University, Durham, United Kingdom

83: Also at Monash University, Faculty of Science, Clayton, Australia

84: Also at Università di Torino, Torino, Italy

85: Also at Bethel University, St. Paul, Minnesota, U.S.A.

86: Also at Karamanoğlu Mehmetbey University, Karaman, Turkey

87: Also at Bingol University, Bingol, Turkey

88: Also at Georgian Technical University, Tbilisi, Georgia

89: Also at Sinop University, Sinop, Turkey

90: Also at Erciyes University, Kayseri, Turkey

91: Also at Texas A\&M University at Qatar, Doha, Qatar

92: Also at Kyungpook National University, Daegu, Korea, Daegu, Korea 